\title{
Discovering Hook Length Formulas by an Expansion Technique
}

\author{
Guo-Niu Han \\ I.R.M.A. UMR 7501, Université Louis Pasteur et CNRS \\ 7, rue René-Descartes, F-67084 Strasbourg, France \\ guoniu@math.u-strasbg.fr
}

Submitted: May 13, 2008; Accepted: Oct 10, 2008; Published: Oct 20, 2008
Mathematics Subject Classifications: 05A15, 05A30, 05E15, 05C05

\begin{abstract}
We introduce a hook length expansion technique and explain how to discover old and new hook length formulas for partitions and plane trees. The new hook length formulas for trees obtained by our method can be proved rather easily, whereas those for partitions are much more difficult and some of them still remain open conjectures. We also develop a Maple package HookExp for computing the hook length expansion. The paper can be seen as a collection of hook length formulas for partitons and plane trees. All examples are illustrated by HookExp and, for many easy cases, expained by well-known combinatorial arguments.
\end{abstract}

\section{Summary}

$\S 1$. Introduction. Selected hook formulas. Conjecture

$\S 2$. Classical hook length formulas for partitions.

§3. Hook length expansion algorithm and HookExp.

$\S 4$. The exponent principle.

$\S 5$. Hook length formulas for partitions.

$\S 6$. Hook length formulas for binary trees.

$\S 7$. Hook length formulas for complete binary trees.

$\S 8$. Hook length formulas for Fibonacci trees.

\section{Introduction}

The hook lengths for partitions and plane trees play an important role in Enumerative Combinatorics. The classical hook length formulas for those two structures read

$$
f_{\lambda}=\frac{n !}{\prod_{v \in \lambda} h_{v}} \quad \text { and } \quad f_{T}=\frac{n !}{\prod_{v \in T} h_{v}},
$$

where $f_{\lambda}$ (resp. $f_{T}$ ) is the number of standard Young tableaux of shape $\lambda$ (resp. of increasing labeled binary trees of shape $T$ ). See Sections 2 and 6 for notations and 
explanations. From the above formulas we can derive

$$
\sum_{\lambda \in \mathcal{P}} x^{|\lambda|} \prod_{v \in \lambda} \frac{1}{h_{v}^{2}}=e^{x}
$$

and

$$
\sum_{T \in \mathcal{B}} x^{|T|} \prod_{v \in T} \frac{1}{h_{v}}=\frac{1}{1-x} .
$$

Formulas (1.1) and (1.2) are referred to as the basic hook length formulas, or hook formulas, for short.

The numerous extensions or generalizations which have been proposed in the literature led us to believe that a technical tool had to be constructed that would make it possible to discover new hook length formulas and also obtain the old ones in a systematic manner. The purpose of this paper is to present such a tool that will be called hook length expansion technique. In general, the new hook length formulas for trees produced by that technique can be proved easily, whereas those for partitions are much more difficult and some of them still remain open conjectures.

We also develop a Maple package HookExp for computing the hook length expansion, which can be downloaded freely from the author's web site. ${ }^{(*)}$ All the examples in the paper are illustrated by HookExp and, for many easy cases, explained by well-known combinatorial arguments.

Sections 2-5 are devoted to the hook length formulas for partitions and Sections 68 for plane trees. Basic notions and classical hook length formulas for partitions are recalled in Section 2. Then, we introduce the hook length expansion algorithm for partitions. In Section 4 we discuss some techniques for discovering new hook length formulas, namely the exponent principle. The new hook formulas for partitions $\lambda \in \mathcal{P}$ (resp. for binary trees $T \in \mathcal{B}$, for complete binary trees $T \in \mathcal{C}$, for Fibonacci trees $T \in \mathcal{F}$ ) were suggested (but not proved!) by playing with the package HookExp. They are all collected in Section 5 (resp. Section 6, 7, 8). The formulas we should like to single out are stated next. See Sections 5-8 for notations, comments and/or proofs.

Theorem $1.1[=5.5]$. Let $t$ be a positive integer and $\operatorname{hmul}_{t}(\lambda)$ be the number of boxes $v$ such that $h_{v}(\lambda)$ is a multiple of $t$. Then

$$
\sum_{\lambda \in \mathcal{P}} x^{|\lambda|}(-1)^{\mathrm{hmul}_{t}(\lambda)}=\prod_{k \geq 1} \frac{\left(1-x^{4 t k}\right)^{t}\left(1-x^{t k}\right)^{2 t}}{\left(1-x^{2 t k}\right)^{3 t}\left(1-x^{k}\right)} .
$$

Theorem $1.2[=5.9]$. We have

$$
\sum_{\lambda \in \mathcal{P}} x^{|\lambda|} \prod_{v \in \lambda}\left(1-\frac{2}{h_{v}^{2}}\right)=\prod_{k \geq 1}\left(1-x^{k}\right) .
$$

$(*)$ Hook length formula homepage

http://math.u-strasbg.fr/ guoniu/hook 
Theorem $1.3[=5.8]$. We have

$$
\sum_{\lambda \in \mathcal{P}} x^{|\lambda|} \prod_{v \in \lambda, h_{v} \text { even }}\left(1-\frac{2}{h_{v}^{2}}\right)=\prod_{k \geq 1}\left(1+x^{k}\right) .
$$

The most general form of the above three theorems is Theorem 5.7. In fact, the latter theorem unifies several formulas, including the Jacobi triple product identity, the Macdonald identities for $A_{\ell}^{(a)}$, the generating functions for partitions (2.5) and for $t$-cores (5.4), the Nekrasov-Okounkov identity (5.1), Theorems 5.3-5.6 and Theorems 1.2-1.3. See $[\mathrm{Ha} 08 \mathrm{e}]$ for the proof and applications of Theorem 5.7.

Conjecture $1.4[=5.2]$. We have

$$
\sum_{\lambda \in \mathcal{P}} x^{|\lambda|} \prod_{v \in \lambda} \rho\left(z ; h_{v}\right)=e^{x+z x^{2} / 2}
$$

where the weight function $\rho(z ; n)$ is defined by

$$
\rho(z ; n)=\frac{\sum_{k=0}^{\lfloor n / 2\rfloor}\left(\begin{array}{c}
n \\
2 k
\end{array}\right) z^{k}}{n \sum_{k=0}^{\lfloor(n-1) / 2\rfloor}\left(\begin{array}{c}
n \\
2 k+1
\end{array}\right) z^{k}} .
$$

Theorem $1.5[=6.3]$. We have

$$
\sum_{T \in \mathcal{B}} x^{|T|} \prod_{v \in T} \frac{1}{h_{v} 2^{h_{v}-1}}=e^{x}
$$

Theorem $1.6[=6.6]$. We have

$$
\sum_{T \in \mathcal{B}} x^{|T|} \prod_{v \in T} \frac{6}{h_{v}\left(h_{v}+2\right)}=\frac{1}{(1-x)^{2}} .
$$

Theorem $1.7[=6.7]$. We have

$$
\sum_{T \in \mathcal{B}} x^{|T|} \prod_{v \in T} \frac{h_{v}+3}{2 h_{v}}=\left(\frac{1-\sqrt{1-4 x}}{2 x}\right)^{2} .
$$

The most general form of Theorem 1.7 is Theorem 6.8. In fact, the latter theorem unifies a lot of formulas, including the two classical hook formulas (6.4) and (6.5), Postnikov's formula (6.7) and the generalization due to Lascoux, Du and Liu, another generalization of Postnikov's formula (6.10), Theorems 6.6 and 6.7. 
Theorem $1.8[=7.2]$. We have

$$
\sum_{T \in \mathcal{C}} x^{|T|} \prod_{v \in T, h_{v} \geq 2} \frac{1}{h_{v} 2^{h_{v}-2}}=e^{x} .
$$

Theorem $1.9[=\mathbf{8 . 6}]$. We have

$$
\sum_{T \in \mathcal{F}} x^{|T|} \prod_{v \in T, h_{v} \geq 2} \frac{4\left(2 h_{v}-1\right)\left(2 h_{v}-3\right)}{\left(h_{v}+1\right)\left(5 h_{v}-6\right)}=\frac{1-\sqrt{1-4 x}}{2 x} .
$$

To clarify the nature of the paper we end the introduction by insisting on the following facts.

1. We introduce the hook length expansion technique by means of an explicit algorithm (Algorithm 3.1), together with the maple package HookExp.

2. The package HookExp is used to compute the first values of the weight functions, which, in principle, suggest hook formulas to human mathematicians. The package itself does not output hook formulas, it does not prove hook formulas either!

3. We list new formulas found by HookEx, but also some known formulas.

4. Most of the hook formulas for partitions are listed without any proofs. Instead, we give the references containing the proofs, usually difficult and lengthy.

5. Most of the hook formulas for binary trees are listed with proofs, even for wellknown formulas, because most of them are proved in a unified way.

6. Sometimes special cases of a master formula are also given, because they have simpler forms with fewer parameters and show how the master formula was found by the author.

\section{Classical hook length formulas for partitions}

The basic notions needed here can be found in [Ma95, p.1; St99, p.287; La01, p.1; Kn98, p.59; An76, p.1]. A partition $\lambda$ is a sequence of positive integers $\lambda=$ $\left(\lambda_{1}, \lambda_{2}, \cdots, \lambda_{\ell}\right)$ such that $\lambda_{1} \geq \lambda_{2} \geq \cdots \geq \lambda_{\ell}>0$. The integers $\left(\lambda_{i}\right)_{i=1,2, \ldots, \ell}$ are called the parts of $\lambda$, the number $\ell$ of parts being the length of $\lambda$ denoted by $\ell(\lambda)$. The sum of its parts $\lambda_{1}+\lambda_{2}+\cdots+\lambda_{\ell}$ is denoted by $|\lambda|$. Let $n$ be an integer. A partition $\lambda$ is said to be a partition of $n$ if $|\lambda|=n$. We write $\lambda \vdash n$. The set of all partitions of $n$ is denoted by $\mathcal{P}(n)$. The set of all partitions is denoted by $\mathcal{P}$, so that

$$
\mathcal{P}=\bigcup_{n \geq 0} \mathcal{P}(n)
$$

Each partition can be represented by its Ferrers diagram. For example, $\lambda=(6,3,3,2)$ is a partition and its Ferrers diagram is reproduced in Fig. 2.1.

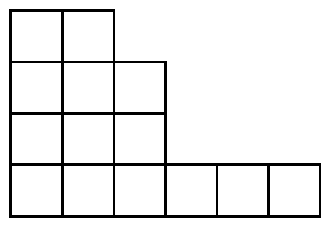

Fig. 2.1. Partition

\begin{tabular}{|c|c|c|c|c|}
\hline 2 & 1 & & & \\
\hline 4 & 3 & 1 & & \\
\hline 5 & 4 & 2 & & \\
\hline 9 & 8 & 6 & & 2 \\
\hline
\end{tabular}

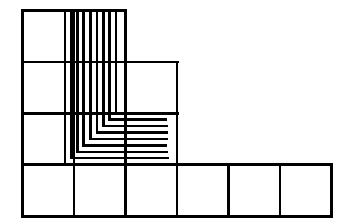

Fig. 2.2. Hook length
Fig. 2.3. Hook lengths 
For each box $v$ in the Ferrers diagram of a partition $\lambda$, or for each box $v$ in $\lambda$, for short, define the hook length of $v$, denoted by $h_{v}(\lambda)$ or $h_{v}$, to be the number of boxes $u$ such that $u=v$, or $u$ lies in the same column as $v$ and above $v$, or in the same row as $v$ and to the right of $v$ (see Fig. 2.2). The hook length multi-set of $\lambda$, denoted by $\mathcal{H}(\lambda)$, is the multi-set of all hook lengths of $\lambda$. In Fig. 2.3 the hook lengths of all boxes for the partition $\lambda=(6,3,3,2)$ have been written in each box. We have $\mathcal{H}(\lambda)=\{2,1,4,3,1,5,4,2,9,8,6,3,2,1\}$. The hook length plays an important role in Algebraic Combinatorics thanks to the famous hook formula due to Frame, Robinson and Thrall [FRT54]

$$
f_{\lambda}=\frac{n !}{\prod_{h \in \mathcal{H}(\lambda)} h},
$$

where $f_{\lambda}$ is the number of standard Young tableaux of shape $\lambda$ (see [St99, p.376; Kn98, p.59; GNW79; RW83; Ze84; GV85; NPS97; Kr99]).

Recall that the Robinson-Schensted-Knuth correspondence (see, for example, [Kn98, p.49-59; St99, p.324]) is a bijection between the set of ordered pairs of standard Young tableaux of $\{1,2, \ldots, n\}$ of the same shape and the set of permutations of order $n$. It provides a combinatorial proof of the following identity.

$$
\sum_{\lambda \vdash n} f_{\lambda}^{2}=n !
$$

By using (2.1) identity (2.2) can be written in the following generating function form

$$
\sum_{\lambda \in \mathcal{P}} x^{|\lambda|} \prod_{h \in \mathcal{H}(\lambda)} \frac{1}{h^{2}}=e^{x} .
$$

The Robinson-Schensted-Knuth correspondence also proves the fact that the number of standard Young tableaux of $\{1,2, \ldots, n\}$ is equal to the number of involutions of order $n$ (see $[\mathrm{Kn} 98 \mathrm{~b}, \mathrm{p} .47 ; \mathrm{Sch} 76]$ ). In the generating function form this means that

$$
\sum_{\lambda \in \mathcal{P}} x^{|\lambda|} \prod_{h \in \mathcal{H}(\lambda)} \frac{1}{h}=e^{x+x^{2} / 2} .
$$

The following identity is the well-known formula for the generating function for partitions [An76, p.3].

$$
\sum_{\lambda \in \mathcal{P}} x^{|\lambda|} \prod_{h \in \mathcal{H}(\lambda)} 1=\prod_{k \geq 1} \frac{1}{1-x^{k}}
$$

In the present paper formulas (2.3), (2.4) and (2.5) are also called hook formulas. We will find other hook formulas in the next sections. 


\section{Hook length expansion algorithm and HookExp}

To express our main algorithm in a handy manner it is convenient to introduce the following definition.

Definition 3.1. Let $\rho: \mathbb{N}^{*} \rightarrow K$ be a map of the set of positive integers to some field $K$. Also let $f(x) \in K[[x]]$ be a formal power series in $x$ with coefficients in $K$ such that $f(0)=1$. If

$$
\sum_{\lambda \in \mathcal{P}} x^{|\lambda|} \prod_{h \in \mathcal{H}(\lambda)} \rho(h)=f(x),
$$

the series $f(x)$ is called the generating function for partitions by the weight function $\rho$. The left-hand side of (3.1) is called the hook length expansion of $f(x)$. Furthermore, when both $\rho$ and $f(x)$ have simple (some people say "nice") forms, equation (3.1) is called a hook length formula, or hook formula for short.

It is easy to see that the generating function $f(x)$ is uniquely determined by the weight function $\rho$. Conversely, the weight function $\rho$ can be uniquely determined by $f(x)$ in most cases. In the other cases (called singular cases), the weight function $\rho$ does not exist, or is not unique. We next provide an algorithm for computing $\rho$ when $f(x)$ is given.

Let $\mathcal{P}_{L}(n)$ be the set of partitions $\lambda=\left(\lambda_{1}, \lambda_{2}, \ldots, \lambda_{\ell}\right)$ of $n$ such that $\ell(\lambda)=1$ or $\lambda_{2}=1$. The partitions in $\mathcal{P}_{L}(n)$ are usually called hooks. The hook length multi-set $\mathcal{H}(\lambda)$ of a hook $\lambda$ of $n$ is simply

$$
\mathcal{H}(\lambda)=\{1,2, \cdots \ell(\lambda)-1,1,2, \cdots, n-\ell(\lambda), n\} .
$$

Let $\mathcal{P}_{Z}(n)$ be the set of partitions $\lambda=\left(\lambda_{1}, \lambda_{2}, \ldots, \lambda_{\ell}\right)$ of $n$ such that $\ell \geq 2$ and $\lambda_{2} \geq 2$. It is easy to see that the hook length multi-set of each partition of $\mathcal{P}_{Z}(n)$ does not contain the integer $n$. Since $\mathcal{P}(n)=\mathcal{P}_{L}(n) \cup \mathcal{P}_{Z}(n)$ we have

$$
\begin{aligned}
\sum_{\lambda \vdash n} \prod_{h \in \mathcal{H}(\lambda)} \rho(h) & =\sum_{\lambda \in \mathcal{P}_{L}(n)} \prod_{h \in \mathcal{H}(\lambda)} \rho(h)+\sum_{\lambda \in \mathcal{P}_{Z}(n)} \prod_{h \in \mathcal{H}(\lambda)} \rho(h) \\
& =\rho(n) \sum_{\lambda \in \mathcal{P}_{L}(n)} \prod_{h=1}^{\ell(\lambda)-1} \rho(h) \prod_{h=1}^{n-\ell(\lambda)} \rho(h)+\sum_{\lambda \in \mathcal{P}_{Z}(n)} \prod_{h \in \mathcal{H}(\lambda)} \rho(h) .
\end{aligned}
$$

The weight function $\rho$ can be obtained by the following algorithm.

Algorithm 3.1. Let $f(x)=1+f_{1} x+f_{2} x^{2}+f_{3} x^{3}+\cdots$ be a power series in $x$. The weight function $\rho$ in the hook length expansion of $f(x)$ can be calculated in the following manner. First, let $\rho(1)=f_{1}$. Then, let $n \geq 2$ and suppose that all values $\rho(k)$ for $1 \leq k \leq n-1$ are known and satisfy the following condition

$$
D:=\sum_{\lambda \in \mathcal{P}_{L}(n)} \prod_{h=1}^{\ell(\lambda)-1} \rho(h) \prod_{h=1}^{n-\ell(\lambda)} \rho(h) \neq 0 .
$$


Then, by iteration, $\rho(n)$ is given by

$$
\rho(n)=\frac{f_{n}-\sum_{\lambda \in \mathcal{P}_{Z}(n)} \prod_{h \in \mathcal{H}(\lambda)} \rho(h)}{D} .
$$

We only consider the power series $f(x)$ for which condition (3.4) holds. This is true in most cases. If for some reason condition (3.4) fails to be true, we try to find an extension of $f(x)$ to avoid the singularity. More precisely, we try to find a series $F(x, t) \in K[[t]][[x]]$ such that $f(x)=F(x, 0)$ and condition (3.4) holds for $F(x, t)$ (see (M.5.3) and (M.5.4) for an example).

The Maple package HookExp is developed for computing the first terms of the generating function $f(x)$ and the first values $\rho(n)$ in the hook length expansion. The underlying variable of the series is always $x$. The input format for $f(x)$ is any valid expression in Maple and the output format for $f(x)$ is

$$
1+f_{1} x+f_{2} x^{2}+f_{3} x^{3}+f_{4} x^{4}+\cdots+f_{n} x^{n} .
$$

The input and output formats for $\rho(n)$ are the list

$$
[\rho(1), \rho(2), \rho(3), \ldots, \rho(n)]
$$

The procedure hookgen(rho) computes the generating function $f(x)$ for the given weight function $\rho$, while the procedure hookexp (f, n) computes the weight function $\rho(k)$ for $k=1,2, \ldots, n$. For example, let us verify identity (2.3) by using the HookExp package.

$>$ read("HookExp.mpl"):
> hooktype:="PA": \# working on partitions
$>$ hookexp(exp(x), 8);
\[ \left[1, \frac{1}{4}, \frac{1}{9}, \frac{1}{16}, \frac{1}{25}, \frac{1}{36}, \frac{1}{49}, \frac{1}{64}\right] \]
$>$ hookgen (\%);
\[ 1+x+\frac{1}{2} x^{2}+\frac{1}{6} x^{3}+\frac{1}{24} x^{4}+\frac{1}{120} x^{5}+\frac{1}{720} x^{6}+\frac{1}{5040} x^{7}+\frac{1}{40320} x^{8} \]

Next, verify identities (2.4) and (2.5). 
$>\operatorname{hookexp}\left(\exp \left(x+x^{\wedge} 2 / 2\right), 8\right)$;

$$
\left[1, \frac{1}{2}, \frac{1}{3}, \frac{1}{4}, \frac{1}{5}, \frac{1}{6}, \frac{1}{7}, \frac{1}{8}\right]
$$

$>$ hookgen $(\%)$;

$$
1+x+x^{2}+\frac{2}{3} x^{3}+\frac{5}{12} x^{4}+\frac{13}{60} x^{5}+\frac{19}{180} x^{6}+\frac{29}{630} x^{7}+\frac{191}{10080} x^{8}
$$

$>\operatorname{hookexp}\left(\operatorname{product}\left(1 /\left(1-\mathrm{x}^{\wedge} \mathrm{k}\right), \mathrm{k}=1 \ldots 9\right), 9\right)$;

$$
[1,1,1,1,1,1,1,1,1]
$$

$>\operatorname{hookgen}(\%)$;

$$
1+x+2 x^{2}+3 x^{3}+5 x^{4}+7 x^{5}+11 x^{6}+15 x^{7}+22 x^{8}+30 x^{9}
$$

\section{The exponent principle}

In principle, the HookExp package gives rise to "millions" of hook expansions. But experience shows that only few of them can be duly named formulas. For example, with the very simple function $1 /(1-x)$, we get the following expansion.

$$
\begin{aligned}
& >\operatorname{hookexp}(1 /(1-\mathrm{x}), 8) ; \\
& \qquad\left[1, \frac{1}{2}, \frac{1}{2}, \frac{7}{12}, \frac{17}{25}, \frac{447}{592}, \frac{160933}{197641}, \frac{105940688107}{124616941064}\right]
\end{aligned}
$$

Apparently, no simple form can be obtained for $\rho(n)$. Next, try to expand the generating function for the famous Catalan numbers (see, e.g., [St99, p.220]).

$>\operatorname{hookexp}((1-\operatorname{sqrt}(1-4 * x)) /(2 * x), 8)$; (M.4.2)

$$
\left[1,1, \frac{5}{3}, \frac{37}{16}, \frac{823}{289}, \frac{85028}{28605}, \frac{1055952653}{323028029}\right]
$$

Not lucky again.

Then, consider the generating function $f(x)$ for the given weight function $\rho(n)=$ $1+1 / n$. 
$>\operatorname{hookgen}([\operatorname{seq}(1+1 / \mathrm{n}, \mathrm{n}=1 . .8)]) ;$

$$
1+2 x+6 x^{2}+\frac{40}{3} x^{3}+31 x^{4}+62 x^{5}+\frac{647}{5} x^{6}+\frac{3664}{15} x^{7}+\frac{98467}{210} x^{8}
$$

No evident formula for $f(x)$. Those three examples tell us that it is not easy to discover hook formulas even with the help of HookExp. In fact, the author derived Algorithm 3.1 a long time ago, but never found any new hook length formula, until he recently discovered the following exponent principle.

The Exponent Principle. If the power series $f(x)$ has a "nice" hook length expansion, then there is a good chance that $f^{z}(x)$ also has a "nice" hook length expansion.

The exponent principle was first discovered for binary trees. In such a case the exponent principle can be partially justified (see (6.3)). It is then successfully applied for finding new hook length formulas for partitions. The exponent principle for partitions has been verified by experimental observation. However, the author has no mathematical argument for proving or even partially explaning it.

Let us illustrate the exponent principle with the exponential function (see identity $(2.3))$.

$>$ hookexp $(\exp (z * x), 8)$; (M.4.4)

$$
\left[z, \frac{z}{4}, \frac{z}{9}, \frac{z}{16}, \frac{z}{25}, \frac{z}{36}, \frac{z}{49}, \frac{z}{64}\right]
$$

It means that the following hook length expansion

$$
\sum_{\lambda \in \mathcal{P}} x^{|\lambda|} \prod_{h \in \mathcal{H}(\lambda)} \frac{z}{h^{2}}=e^{z x}
$$

holds, but it is nothing new. We simply recover (2.3). In the next section new hook length formulas for partitions will be derived.

\section{Hook length formulas for partitions}

Let us apply the exponent principle to identity (2.5).

$$
\begin{aligned}
& >\text { hookexp }\left(\operatorname{product}\left(1 /\left(1-\mathrm{x}^{\wedge} \mathrm{k}\right)^{\wedge} \mathrm{z}, \mathrm{k}=1 \ldots 7\right), 7\right) ; \\
& {\left[z, \frac{z+3}{4}, \frac{z+8}{9}, \frac{z+15}{16}, \frac{z+24}{25}, \frac{z+35}{36}, \frac{z+48}{49}\right]}
\end{aligned}
$$

From the above expansion we derive the following hook length formula for the power of Euler Product. 
Theorem 5.1 [Nekrasov-Okounkov]. For any complex number $\beta$ we have

$$
\sum_{\lambda \in \mathcal{P}} \prod_{h \in \mathcal{H}(\lambda)}\left(1-\frac{\beta}{h^{2}}\right) x=\prod_{k \geq 1}\left(1-x^{k}\right)^{\beta-1} .
$$

Theorem 5.1 was discovered by Nekrasov and Okounkov in the study of the SeibergWitten Theory [NO06, arXiv:hep-th/0306238v2, formula (6.12), p.55]. In an unpublished paper (available on arXiv [Ha08a]) the author re-discovered the Nekrasov-Okounkov identity (5.1) and gave an elementary proof by using the Macdonald identities [Ma72]. Several applications were also derived, including the marked hook formula.

Now consider identity (2.4). The series

$$
f(x)=e^{x+x^{2} / 2}
$$

is the generating function for involutions. By the exponent principle there is a good chance that

$$
f^{z}(x)=e^{z x+z x^{2} / 2} \quad \text { or } \quad f^{1 / z}(z x)=e^{x+z x^{2} / 2}
$$

has a "nice" hook length expansion.

$$
\begin{aligned}
& >\text { hookexp }\left(\exp \left(x+z * x^{\wedge} 2 / 2\right), 9\right) \text {; } \\
& {\left[1, \frac{1+z}{4}, \frac{3 z+1}{9+3 z}, \frac{z^{2}+6 z+1}{16+16 z}, \frac{5 z^{2}+10 z+1}{5 z^{2}+50 z+25}, \frac{z^{3}+15 z^{2}+15 z+1}{120 z+36 z^{2}+36},\right.} \\
& \left.\frac{7 z^{3}+35 z^{2}+21 z+1}{7 z^{3}+147 z^{2}+245 z+49}, \frac{z^{4}+28 z^{3}+70 z^{2}+28 z+1}{448 z^{2}+64 z^{3}+448 z+64}\right]
\end{aligned}
$$

The above values of $\rho$ suggests that the following new hook length formula, seen as an interpolation between permutations (2.3) and involutions (2.4), should hold.

Conjecture 5.2. We have

$$
\sum_{\lambda \in \mathcal{P}} x^{|\lambda|} \prod_{h \in \mathcal{H}(\lambda)} \rho(z ; h)=e^{x+z x^{2} / 2}
$$

where the weight function $\rho(z ; n)$ is defined by

$$
\rho(z ; n)=\frac{\sum_{k=0}^{\lfloor n / 2\rfloor}\left(\begin{array}{c}
n \\
2 k
\end{array}\right) z^{k}}{n \sum_{k=0}^{\lfloor(n-1) / 2\rfloor}\left(\begin{array}{c}
n \\
2 k+1
\end{array}\right) z^{k}} .
$$


When $z=1$, then $\rho(1 ; n)=1 / n$. Identity (5.2) is true thanks to identity (2.4). When $z=0$, then $\rho(0 ; n)=1 / n^{2}$. Identity (5.2) is also true, since it becomes identity (2.3). However, we cannot prove any other special cases of Conjecture 5.2, except the above two values. For more remarks about Conjecture 5.2, see [Ha08d].

Recall that a partition $\lambda$ is a $t$-core if the hook length multi-set of $\lambda$ does not contain the integer $t$. It is known that the hook length multi-set of each $t$-core does not contain any multiple of $t$. The generating function for $t$-cores is given by the following formula:

$$
\sum_{\lambda} x^{|\lambda|}=\prod_{k \geq 1} \frac{\left(1-x^{t k}\right)^{t}}{1-x^{k}}
$$

where the sum ranges over all $t$-cores [Kn98. p.69, p.612; St99, p.468; GKS90].

Why do not expand the right-hand side of (5.4) by using HookExp? There is an interesting history hidden behind formula (5.4). Since $t$ is a positive integer but not a free parameter, we must choose a numerical value for $t$. Take $t=3$, formula (5.4) says that $\rho$ must have the following form

$$
[1,1,0,1,1, *, 1,1, *, 1,1, *],
$$

where $*$ can be any numerical number.

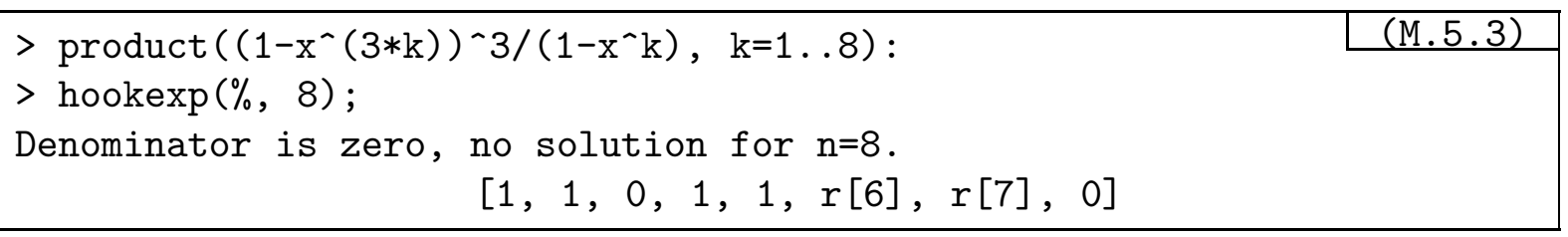

We cannot obtain (5.5) directly by using HookExp. It is a sigular case. To avoid the sigularity we replace the 3 in the exponent by a free parameter $z$ (see comments after Algorithm 3.1). Now hookexp does not report any error message. Finally we replace $z$ by 3 to recover the $\rho$ shown in (5.5) (see also (M.5.12) for another variation of (M.5.3)).

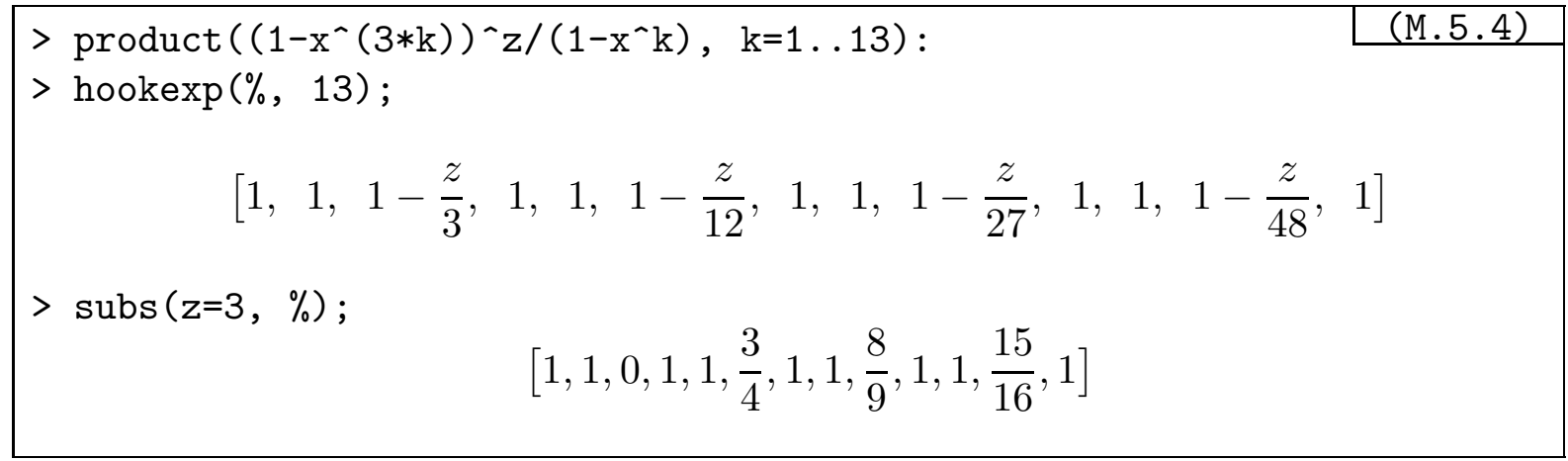

Moreover, the above expansion suggests the following hook length formula, which may be seen as an interpolation between identity (5.4) and a specialization of (5.1):

$$
\sum_{\lambda} x^{|\lambda|} \prod_{v \in \lambda}\left(1-\frac{t^{2}}{h_{v}^{2}}\right)=\prod_{k \geq 1} \frac{\left(1-x^{k}\right)^{t^{2}}}{1-x^{k}} .
$$


Theorem 5.3. We have the following hook length formula

$$
\sum_{\lambda \in \mathcal{P}} x^{|\lambda|} \prod_{h \in \mathcal{H}(\lambda)} \rho(z ; h)=\prod_{k \geq 1} \frac{\left(1-x^{t k}\right)^{z}}{1-x^{k}}
$$

where the weight function $\rho(z ; n)$ is defined by

$$
\rho(z ; n)= \begin{cases}1 ; & \text { if } n \not \equiv 0 \bmod t \\ 1-\frac{t z}{n^{2}} ; & \text { if } n \equiv 0 \bmod t .\end{cases}
$$

When $z=t$ we recover identity (5.4). When $t=1$ and $z=t^{2}$ we recover identity (5.6). Theorem 5.3 is a special case of Theorem 5.7, which is proved in [Ha08e].

Now let us verify Theorem 5.3 by using hookgen for $t=2$ instead of hookexp. In addition of HookExp, we also use the Maple package qseries developed by Frank Garvan [Ga01]. Recall that the Dedekind $\eta$-function, is defined by $\eta(x)=x^{1 / 24} \prod_{m \geq 0}\left(1-x^{m}\right)$.

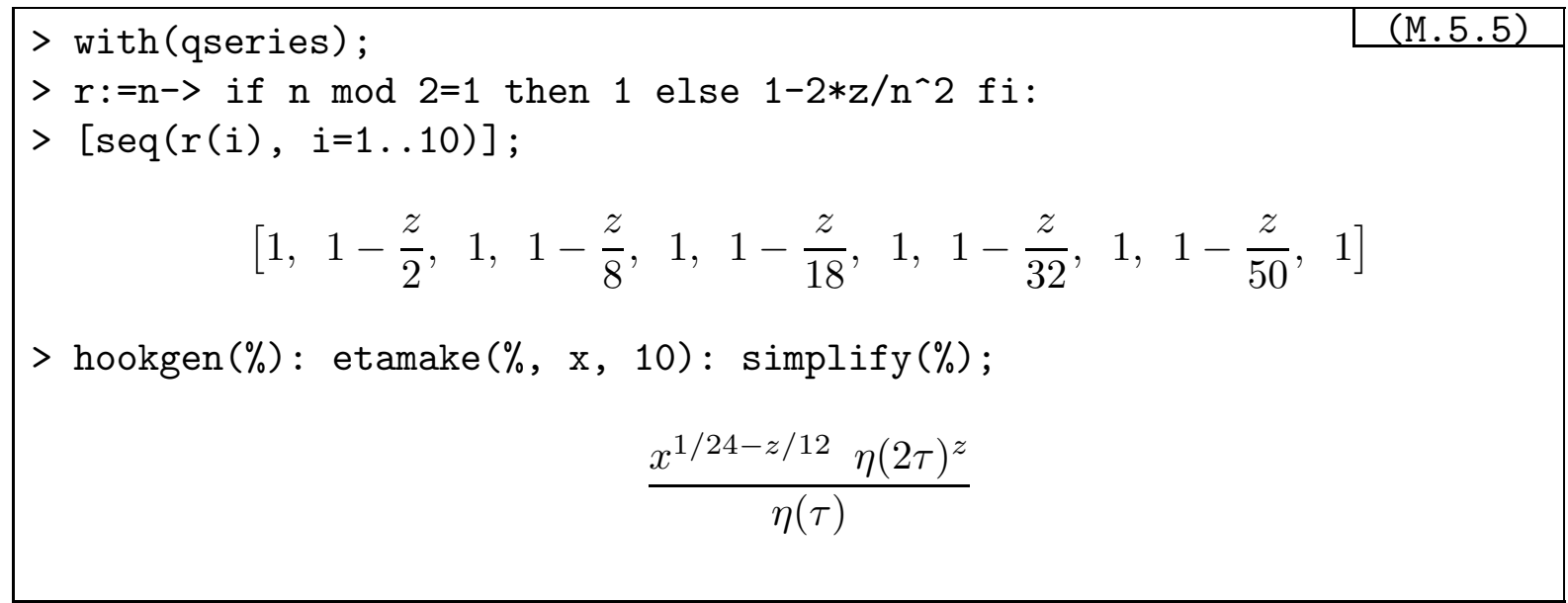

As expected we obtain the right-hand side of (5.7) for $t=2$. Next we hope to obtain new hook formula by modifying slightly the above weight function. Try to change the 1 in odd position by -1 .

$$
\begin{aligned}
& >\mathrm{r}:=\mathrm{n}->\text { if } \mathrm{n} \bmod 2=1 \text { then }-1 \text { else } 1-2 * \mathrm{z} / \mathrm{n}^{\wedge} 2 \mathrm{fi}: \\
& >[\operatorname{seq}(\mathrm{r}(\mathrm{i}), \mathrm{i}=1 \ldots 10)] ; \\
& \quad\left[-1,1-\frac{z}{2},-1,1-\frac{z}{8},-1,1-\frac{z}{18},-1,1-\frac{z}{32},-1,1-\frac{z}{50},-1\right] \\
& >\operatorname{hookgen}(\%): \operatorname{etamake}(\%, \mathrm{x}, 10): \operatorname{simplify}(\%) ; \\
& x^{1 / 24-z / 12} \eta(8 \tau)^{2-z} \eta(4 \tau)^{-5+3 z} \eta(2 \tau)^{1-z} \eta(\tau)
\end{aligned}
$$

The above expansion suggests the following hook length formula for partitions. 
Theorem 5.4. We have the following hook length formula

$$
\sum_{\lambda \in \mathcal{P}} x^{|\lambda|} \prod_{h \in \mathcal{H}(\lambda)} \rho(z ; h)=\prod_{k \geq 1} \frac{\left(1-x^{k}\right)\left(1-x^{4 k}\right)^{3 z-5}}{\left(1-x^{8 k}\right)^{z-2}\left(1-x^{2 k}\right)^{z-1}} .
$$

where the weight function $\rho(z ; n)$ is defined by

$$
\rho(z ; n)= \begin{cases}-1 ; & \text { if } n \neq \equiv \bmod 2 . \\ 1-\frac{2 z}{n^{2}} ; & \text { if } n \equiv 0 \bmod 2 .\end{cases}
$$

Inspired by Theorem 5.4, we calculate the generating function for partitions by the following periodical weight function $\rho$.

$$
\begin{gathered}
>\mathrm{r}:=\mathrm{n}->\text { if } \mathrm{n} \bmod 3=0 \text { then }-1 \text { else } 1 \mathrm{fi}: \\
>[\operatorname{seq}(\mathrm{r}(\mathrm{i}), \mathrm{i}=1 \ldots 17)] ; \\
{[1,1,-1,1,1,-1,1,1,-1,1,1,-1,1,1,-1,1,1]} \\
>\operatorname{hookgen}(\%): \operatorname{etamake}(\%, \mathrm{x}, 17): \operatorname{simplify}(\%) ; \\
\frac{x^{1 / 24} \eta(12 \tau)^{3} \eta(3 \tau)^{6}}{\eta(6 \tau)^{9} \eta(\tau)}
\end{gathered}
$$

The above hook length expansion suggests the following formula.

Theorem $5.5[=\mathbf{1 . 1}]$. Let $t$ be a positive integer and $\operatorname{hmul}_{t}(\lambda)$ be the number of boxes $v$ such that $h_{v}(\lambda)$ is a multiple of $t$. Then

$$
\sum_{\lambda \in \mathcal{P}} x^{|\lambda|}(-1)^{\mathrm{hmul}_{t}(\lambda)}=\prod_{k \geq 1} \frac{\left(1-x^{4 t k}\right)^{t}\left(1-x^{t k}\right)^{2 t}}{\left(1-x^{2 t k}\right)^{3 t}\left(1-x^{k}\right)} .
$$

In fact, Theorem 5.5 can be generalized by replacing -1 by $z$.

$>\mathrm{f}:=\mathrm{k} \rightarrow\left(1-\mathrm{x}^{\wedge}(3 * \mathrm{k})\right)^{\wedge} 3 /\left(1-\left(z * \mathrm{x}^{\wedge} 3\right)^{\wedge} \mathrm{k}\right)^{\wedge} 3 /\left(1-\mathrm{x}^{\wedge} \mathrm{k}\right):$
$>$ hookexp(product $(\mathrm{f}(\mathrm{k}), \mathrm{k}=1 \ldots 15), 15) ;$
\[ [1,1, z, 1,1, z, 1,1, z, 1,1, z, 1,1, z] \]

Theorem 5.6. Let $t$ be a positive integer. Then

$$
\sum_{\lambda \in \mathcal{P}} x^{|\lambda|} z^{\mathrm{hmul}_{t}(\lambda)}=\prod_{k \geq 1} \frac{\left(1-x^{t k}\right)^{t}}{\left(1-\left(z x^{t}\right)^{k}\right)^{t}\left(1-x^{k}\right)} .
$$


We can unify (M.5.4) and (M.5.8) in the following manner.

$>\mathrm{N}:=14: \mathrm{t}:=3:$
$>\mathrm{r}:=\mathrm{n}->$ if $\mathrm{n} \bmod \mathrm{t}=0$ then $\mathrm{y} *\left(1-\mathrm{t} * \mathrm{z} / \mathrm{n}^{\wedge} 2\right)$ else $1 \mathrm{fi}:$
$>\mathrm{fk}:=\mathrm{k}->\left(\left(1-\mathrm{x}^{\wedge}(\mathrm{t} * \mathrm{k})\right)^{\wedge} \mathrm{t}\right) /\left(\left(1-\left(\mathrm{y} * \mathrm{x}^{\wedge} \mathrm{t}\right)^{\wedge} \mathrm{k}\right) \wedge(\mathrm{t}-\mathrm{z})\right) /\left(1-\mathrm{x}^{\wedge} \mathrm{k}\right):$
$>\operatorname{seq}(\mathrm{r}(\mathrm{i}), \mathrm{i}=1 \ldots \mathrm{N})] ;$
$\quad\left[1,1, y-\frac{y z}{3}, 1,1, y-\frac{y z}{12}, 1,1, y-\frac{y z}{27}, 1,1, y-\frac{y z}{48}, 1,1\right]$
$>\operatorname{hookgen}(\%)-\operatorname{product}(\mathrm{fk}(\mathrm{k}), \mathrm{k}=1 \ldots \mathrm{N}):$
$>\operatorname{series}(\%, \mathrm{x}, \mathrm{N}+1): \operatorname{simplify}(\%) ;$
$\quad O\left(x^{15}\right)$

The above expansion suggests the following hook length formula.

Theorem 5.7. We have

$$
\sum_{\lambda \in \mathcal{P}} x^{|\lambda|} \prod_{h \in \mathcal{H}(\lambda)} \rho(z ; h)=\prod_{k \geq 1} \frac{\left(1-x^{t k}\right)^{t}}{\left(1-\left(y x^{t}\right)^{k}\right)^{t-z}\left(1-x^{k}\right)},
$$

where the weight function $\rho(z ; n)$ is defined by

$$
\rho(z ; n)= \begin{cases}1 ; & \text { if } n \neq \equiv 0 \bmod t . \\ y-\frac{t y z}{n^{2}} ; & \text { if } n \equiv 0 \bmod t .\end{cases}
$$

The proof of Theorem 5.7, as well as some applications can be found in [Ha08e]. Let us single out the very simple case when $t=2, y=z=1$.

$>\operatorname{hookexp}\left(\operatorname{product}\left(1+\mathrm{x}^{\wedge} \mathrm{k}, \mathrm{k}=1 \ldots 14\right), 14\right)$;

(M.5.10)

$$
\left[1, \frac{1}{2}, 1, \frac{7}{8}, 1, \frac{17}{18}, 1, \frac{31}{32}, 1, \frac{49}{50}, 1, \frac{71}{72}, 1, \frac{97}{98}\right]
$$

Theorem $5.8[=1.3]$. We have the following hook length formula

$$
\sum_{\lambda \in \mathcal{P}} x^{|\lambda|} \prod_{h \in \mathcal{H}(\lambda), h \text { even }}\left(1-\frac{2}{h^{2}}\right)=\prod_{k \geq 1}\left(1+x^{k}\right) .
$$

The above theorem is to be compared with the following specialization of Theorem 5.7 when $z=2, y=t=1$. 
Theorem $5.9[=1.2]$. We have the following hook length formula

$$
\sum_{\lambda \in \mathcal{P}} x^{|\lambda|} \prod_{h \in \mathcal{H}(\lambda)}\left(1-\frac{2}{h^{2}}\right)=\prod_{k \geq 1}\left(1-x^{k}\right) .
$$

There are also other hook formulas that are not specializations of Theorem 5.7. Consider the weight function $\rho$ that counts the corners (their hook lengths are 1) of partitions.

$$
\begin{aligned}
& >\quad[z, \operatorname{seq}(1, \quad i=1 . .7)] ; \\
& >\operatorname{hookgen}(\%) ; \\
& \quad \begin{array}{l}
1+(z) x+(2 z) x^{2}+\left(2 z+z^{2}\right) x^{3}+\left(3 z+2 z^{2}\right) x^{4}+\left(2 z+5 z^{2}\right) x^{5} \\
\quad+\left(4 z+6 z^{2}+z^{3}\right) x^{6}+\left(2 z+11 z^{2}+2 z^{3}\right) x^{7}+\left(4 z+13 z^{2}+5 z^{3}\right) x^{8}
\end{array}
\end{aligned}
$$

The above generating function corresponds to the sequence A116608 in the on-line Encyclopedia of Integer Sequences [Slo] and is equal to the right-hand side of (5.17) below.

Theorem 5.10. We have

$$
\sum_{\lambda \in \mathcal{P}} x^{|\lambda|} \prod_{h \in \mathcal{H}(\lambda), h=1} z=\prod_{k \geq 1} \frac{1+(z-1) x^{k}}{1-x^{k}} .
$$

On the other hand, take (M.5.3) and change the "_" in the numerator by "+", we get

$>\operatorname{product}\left(\left(1+\mathrm{x}^{\wedge}(3 * \mathrm{k})\right)^{\wedge} 3 /\left(1-\mathrm{x}^{\wedge} \mathrm{k}\right), \mathrm{k}=1 \ldots 8\right)$ :

$>$ hookexp $(\%, 8)$;

$$
[1,1,2,1,1,1,1,1]
$$

The above expansion suggests the following formula.

Theorem 5.11. We have

$$
\sum_{\lambda \in \mathcal{P}} x^{|\lambda|} \prod_{h \in \mathcal{H}(\lambda), h=t} 2=\prod_{k \geq 1} \frac{\left(1+x^{t k}\right)^{t}}{1-x^{k}} .
$$

In [Ha08e] we proved the following unified form of Theorems 5.10 and 5.11 by using the properties of a classical bijection which maps each partition to its $t$-core and t-quotient [Ma95, p.12; St99, p.468; JK81, p.75; GSK90].

Theorem 5.12. For any complex number $z$ we have

$$
\sum_{\lambda \in \mathcal{P}} x^{|\lambda|} \prod_{h \in \mathcal{H}(\lambda), h=t} z=\prod_{k \geq 1} \frac{\left(1+(z-1) x^{t k}\right)^{t}}{1-x^{k}} .
$$




\section{Hook length formulas for binary trees}

The basic notions for binary trees can be found in [St97,p.295; Kn98a, p.308313; Vi81]. A binary tree $T$ with $n=|T|$ vertices is defined recursively as follows. Either $T$ is empty, or else one specially designated vertex $v$ is called the root of $T$; the remaining vertices (excluding the root) are then displayed into an ordered pair $\left(T^{\prime}, T^{\prime \prime}\right)$ of binary trees (possibly empty), called subtrees of the root $v$. The hook length of each vertex $u$, denoted by $h_{u}(T)$ or $h_{u}$, is the number of descendants of $u$ (including $u$ ). Each vertex is called leaf if its two subtrees are both empty. The hook length multi-set $\mathcal{H}(T)=\left\{h_{u} \mid u \in T\right\}$ of $T$ is defined to be the multi-set of hook lengths of all vertices $u$ of $T$. Finally, let $\mathcal{B}($ resp. $\mathcal{B}(n)$ ) denote the set of all binary trees (resp. all binary trees with $n$ vertices), so that

$$
\mathcal{B}=\bigcup_{n \geq 0} \mathcal{B}(n) .
$$

For example, there are five binary trees with $n=3$ vertices.
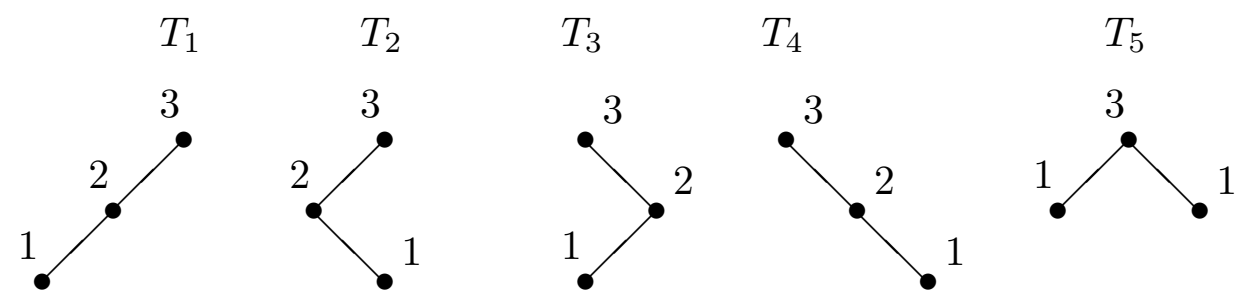

We have $\mathcal{H}\left(T_{1}\right)=\mathcal{H}\left(T_{2}\right)=\mathcal{H}\left(T_{3}\right)=\mathcal{H}\left(T_{4}\right)=\{1,2,3\}$ and $\mathcal{H}\left(T_{5}\right)=\{1,1,3\}$.

As done for the partitions in Definition 3.1 we define the hook length expansion for binary trees by

$$
\sum_{T \in \mathcal{B}} x^{|T|} \prod_{h \in \mathcal{H}(T)} \rho(h)=f(x)
$$

where $f(x) \in K[[x]]$ is a power series in $x$ with coefficients in $K$ such that $f(0)=1$. See Section 3 for other related definitions and comments about hook length expansion. For computing the weight function $\rho$ we need find an analogue of Algorithm 3.1 for binary trees. No surprise, it is much easier to find a formula for computing $\rho$, since the binary tree structure is simple, compared with the partition structure.

Let $f(x)=1+f_{1} x+f_{2} x^{2}+f_{3} x^{3}+\cdots$ be the generating function for binary trees by the weight function $\rho$. With each $T \in \mathcal{B}(n)(n \geq 1)$ we can associate a triplet $\left(T^{\prime}, T^{\prime \prime}, v\right)$, where $T^{\prime} \in \mathcal{B}(k)(0 \leq k \leq n-1), T^{\prime \prime} \in \mathcal{B}(n-1-k)$ and the root $v$ of $T$ whose hook length $h_{v}=n$. Hence (6.1) is equivalent to

$$
\rho(n) \sum_{k=0}^{n-1} f_{k} f_{n-1-k}=f_{n} \quad(n \geq 1) .
$$


Formula (6.2) can be used to calculate $f(x)$ for a given $\rho$, or to calculate $\rho$ for a given $f(x)$. It also has the equivalent form

$$
\rho(n)=\frac{\left[x^{n}\right] f(x)}{\left[x^{n-1}\right] f^{2}(x)},
$$

where $\left[x^{n}\right] f(x)$ means the coefficient of $x^{n}$ in the power series $f(x)$. From (6.3) we may say that finding a hook length formula is equivalent to finding a formal power series $f(x)$ such that $\left[x^{n}\right] f(x) /\left[x^{n-1}\right] f^{2}(x)$ has a "nice" form in $n$.

Next we use the maple package HookExp to find hook formulas for binary trees. The syntax of the two procedures hookexp and hookgen are the same as for partitions. The rest of this section contains some sessions, and each session contains three parts: (i) experiment with HookExp; (ii) hook formula suggested by the experiment; (iii) proof and/or comments of the hook formula. All proofs of the hook formulas presented in this section are always based on relation (6.3).

$$
\begin{aligned}
& >\text { hooktype:="BT": \# working on binary trees } \\
& >\text { hookexp(1/(1-x),9); } \\
& \qquad\left[1, \frac{1}{2}, \frac{1}{3}, \frac{1}{4}, \frac{1}{5}, \frac{1}{6}, \frac{1}{7}, \frac{1}{8}, \frac{1}{9}\right]
\end{aligned}
$$

Theorem 6.1. We have

$$
\sum_{T \in \mathcal{B}} x^{|T|} \prod_{h \in \mathcal{H}(T)} \frac{1}{h}=\frac{1}{1-x} .
$$

Proof. From (6.3)

$$
\rho(n)=\frac{\left[x^{n}\right] 1 /(1-x)}{\left[x^{n-1}\right] 1 /(1-x)^{2}}=1 / n .
$$

Remark. It is well-known [Kn98b,p.67; St75] that the number of ways to label the vertices of $T$ with $\{1,2, \ldots, n\}$, such that the label of each vertex is less than that of its descendants (called increasing labeled binary trees, or labeled binary trees for short), is equal to $n$ ! divided by the product of the $h_{v}$ 's $(v \in T)$. On the other hand, each labeled binary tree with $n$ vertices is in bijection with a permutation of order $n$ [St97,p.24;FS73;Vi81], so that

$$
\sum_{T \in \mathcal{B}(n)} n ! \prod_{v \in T} \frac{1}{h_{v}}=n !
$$

This gives a combinatorial proof of Theorem 6.1.

$>\operatorname{hookexp}((1-\operatorname{sqrt}(1-4 * \mathrm{x})) /(2 * \mathrm{x}), 9)$;

(M.6.2)

$$
[1,1,1,1,1,1,1,1]
$$


Theorem 6.2. We have

$$
\sum_{T \in \mathcal{B}} x^{|T|} \prod_{h \in \mathcal{H}(T)} 1=\frac{1-\sqrt{1-4 x}}{2 x} .
$$

Proof. Let $f(x)$ be the right-hand side of (6.5). Then

$$
\left[x^{n-1}\right] f^{2}(x)=\left[x^{n-1}\right](f(x)-1) / x=\left[x^{n}\right] f(x) . \quad \square
$$

Remark. Formula (6.5) implies that the number of binary trees with $n$ vertices is equal to the $n$-th Catalan number (see, e.g., [St99, p.220])

$$
\sum_{T \in \mathcal{B}(n)} 1=\frac{1}{n+1}\left(\begin{array}{c}
2 n \\
n
\end{array}\right) \text {. }
$$

In the following experiments we make use of the maple package Guess, translated by Béraud and Gauthier [BG04] from the Mathematica package Rate devoloped by Krattenthaler [Kr01].

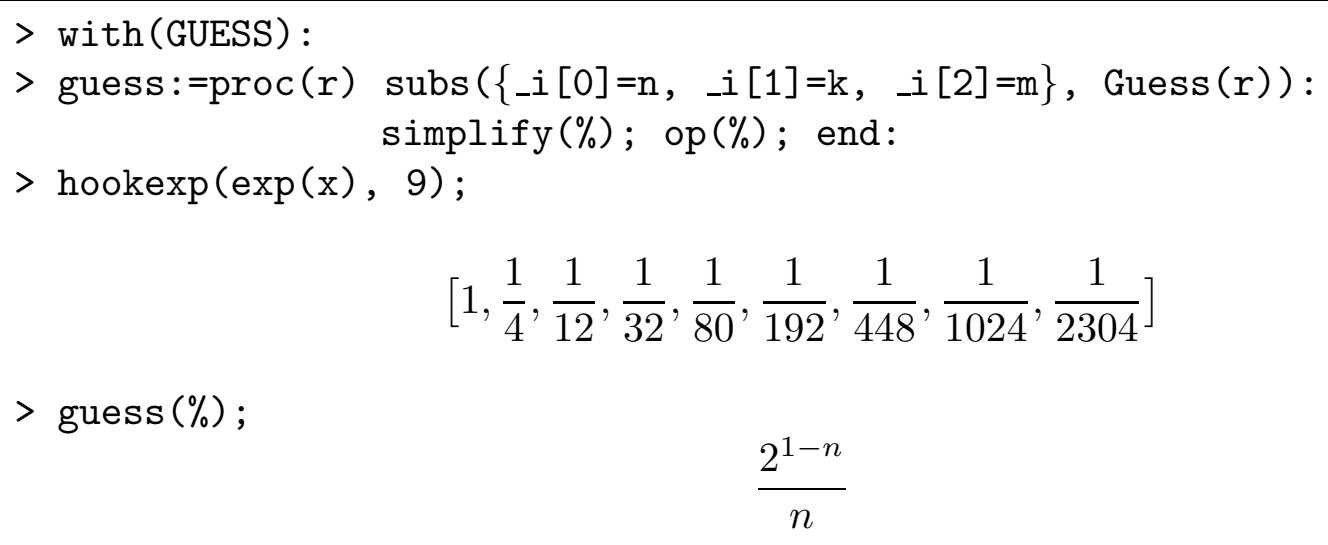$$
\left[1, \frac{1}{4}, \frac{1}{12}, \frac{1}{32}, \frac{1}{80}, \frac{1}{192}, \frac{1}{448}, \frac{1}{1024}, \frac{1}{2304}\right]
$$

$>\operatorname{guess}(\%)$

$$
\frac{2^{1-n}}{n}
$$

Theorem 6.3. We have

$$
\sum_{T \in \mathcal{B}} x^{|T|} \prod_{h \in \mathcal{H}(T)} \frac{1}{h 2^{h-1}}=e^{x}
$$

Proof. By (6.3)

$$
\rho(n)=\frac{\left[x^{n}\right] e^{x}}{\left[x^{n-1}\right] e^{2 x}}=\frac{1 / n !}{2^{n-1} /(n-1) !}=\frac{1}{n 2^{n-1}} .
$$

Remark. We do not have any combinatorial proof of Theorem 6.3. See also [Ha08b]. 
$>[\operatorname{seq}(1+1 / n, n=1 \ldots 7)]$

$$
\left[2, \frac{3}{2}, \frac{4}{3}, \frac{5}{4}, \frac{6}{5}, \frac{7}{6}, \frac{8}{7}\right]
$$

$>\mathrm{f}:=$ hookgen $(\%)$;

$$
1+2 x+6 x^{2}+\frac{64}{3} x^{3}+\frac{250}{3} x^{4}+\frac{1728}{5} x^{5}+\frac{67228}{45} x^{6}+\frac{2097152}{315} x^{7}
$$

Theorem 6.4 [Postnikov]. We have

$$
\sum_{T \in \mathcal{B}} x^{|T|} \prod_{h \in \mathcal{H}(T)}\left(1+\frac{1}{h}\right)=\sum_{n \geq 0}(n+1)^{n-1} \frac{(2 x)^{n}}{n !}
$$

Proof. Let $G(x)$ be a power series such that

$$
G(x)=\exp (x G(x))
$$

By the Lagrange inversion formula $G(x)^{z}$ has the following explicit expansion:

$$
G(x)^{z}=\sum_{n \geq 0} z(n+z)^{n-1} \frac{x^{n}}{n !}
$$

The right-hand side of (6.7) is $G(2 x)$. By (6.3)

$$
\rho(n)=\frac{\left[x^{n}\right] G(2 x)}{\left[x^{n-1}\right] G^{2}(2 x)}=\frac{(n+1)^{n-1} 2^{n} / n !}{2(n+1)^{n-2} 2^{n-1} /(n-1) !}=1+\frac{1}{n} .
$$

Further combinatorial proofs and extensions have been proposed by several authors [Po04, CY08, DL08, GS06, MY07, Se08, Ha08c].

$>[\operatorname{seq}(1+1 / n, n=1 \ldots 9)]$;

$$
\left[2, \frac{3}{2}, \frac{4}{3}, \frac{5}{4}, \frac{6}{5}, \frac{7}{6}, \frac{8}{7}, \frac{9}{8}, \frac{10}{9}\right]
$$

$>f:=$ hookgen $(\%): \operatorname{hookexp}\left(f^{\wedge} z, 7\right): \operatorname{map}($ factor,$\%)$;

$$
\left[2 z, \frac{2+z}{2}, \frac{(z+3)^{2}}{6 z+6}, \frac{(z+4)^{3}}{4(2 z+3)^{2}}, \frac{(z+5)^{4}}{40(2+z)^{3}}, \frac{(z+6)^{5}}{6(2 z+5)^{4}}, \frac{(z+7)^{6}}{224(z+3)^{5}}\right]
$$


Theorem 6.5. We have

$$
\sum_{T \in \mathcal{B}} x^{|T|} \prod_{v \in T} \frac{(z+h)^{h-1}}{h(2 z+h-1)^{h-2}}=\sum_{n \geq 0} z(z+n)^{n-1} \frac{(2 x)^{n}}{n !} .
$$

Proof. From (6.3), (6.8) and (6.9) we have

$$
\begin{aligned}
\rho(n) & =\frac{\left[x^{n}\right] G^{z}(2 x)}{\left[x^{n-1}\right] G^{2 z}(2 x)}=\frac{z(n+z)^{n-1} 2^{n} / n !}{2 z(n+2 z-1)^{n-2} 2^{n-1} /(n-1) !} \\
& =\frac{(z+n)^{n-1}}{n(2 z+n-1)^{n-2}} \cdot
\end{aligned}
$$

Remark. We do not have any combinatorial proof of Theorem 6.5. See also [Ha08c].

$>\operatorname{hookexp}\left(1 /(1-x)^{\wedge} 2,9\right)$;

$$
\left[2, \frac{3}{4}, \frac{2}{5}, \frac{1}{4}, \frac{6}{35}, \frac{1}{8}, \frac{2}{21}, \frac{3}{40}, \frac{2}{33}\right]
$$

$>$ guess $(\%)$;

$$
\frac{6}{n(n+2)}
$$

Theorem 6.6. We have

$$
\sum_{T \in \mathcal{B}} x^{|T|} \prod_{v \in T} \frac{6}{n(n+2)}=\frac{1}{(1-x)^{2}}
$$

More generally,

$$
\sum_{T \in \mathcal{B}} x^{|T|} \prod_{h \in \mathcal{H}(T)} \frac{\prod_{i=1}^{h-1}(z+i)}{2 h \prod_{i=1}^{h-2}(2 z+i)}=\frac{1}{(1-x)^{z}}
$$

or

$$
\sum_{T \in \mathcal{B}(n)} \prod_{h \in \mathcal{H}(T)} \frac{\prod_{i=1}^{h-1}(z+i)}{2 h \prod_{i=1}^{h-2}(2 z+i)}=\frac{1}{n !} \prod_{i=0}^{n-1}(z+i) .
$$

Proof. By (6.3)

$$
\rho(n)=\frac{\left[x^{n}\right] 1 /(1-x)^{z}}{\left[x^{n-1}\right] 1 /(1-x)^{2 z}}=\frac{\left(\begin{array}{c}
n+z-1 \\
n
\end{array}\right)}{\left(\begin{array}{c}
n+2 z-2 \\
n-1
\end{array}\right)} .
$$


$>\operatorname{hookexp}\left(((1-\operatorname{sqrt}(1-4 * x)) /(2 * x))^{\wedge} 2,10\right)$

$$
\left[2, \frac{5}{4}, 1, \frac{7}{8}, \frac{4}{5}, \frac{3}{4}, \frac{5}{7}, \frac{11}{16}\right]
$$

$>\operatorname{guess}(r)$

$$
\frac{n+3}{2 n}
$$

Theorem 6.7. We have

$$
\sum_{T \in \mathcal{B}} x^{|T|} \prod_{h \in \mathcal{H}(T)} \frac{h+3}{2 h}=\left(\frac{1-\sqrt{1-4 x}}{2 x}\right)^{2}
$$

More generally,

$$
\sum_{T \in \mathcal{B}} x^{|T|} \prod_{h \in \mathcal{H}(T)} \frac{\prod_{i=1}^{h-1}(z+2 h-i)}{2 h \prod_{i=1}^{h-2}(2 z+2 h-2-i)}=\left(\frac{1-\sqrt{1-4 x}}{2 x}\right)^{z},
$$

or

$$
\sum_{T \in \mathcal{B}(n)} \prod_{h \in \mathcal{H}(T)} \frac{\prod_{i=1}^{h-1}(z+2 h-i)}{2 h \prod_{i=1}^{h-2}(2 z+2 h-2-i)}=\frac{z}{n !} \prod_{i=1}^{n-1}(2 n-i+z) .
$$

Theorem 6.5, 6.6 and 6.7 can be derived from the next Theorem by taking $a=1$, $a=0$ and $a \rightarrow \infty$, respectively.

$>[\operatorname{seq}(a+1 / n, n=1 \ldots 7)]$;

$$
\left[a+1, a+\frac{1}{2}, a+\frac{1}{3}, a+\frac{1}{4}, a+\frac{1}{5}, a+\frac{1}{6}, a+\frac{1}{7}\right]
$$

$>f:=h o o k g e n(\%): \operatorname{hookexp}\left(f^{\wedge} z, 5\right): \operatorname{map}($ factor,$\%)$;

$$
\begin{aligned}
& {\left[z(a+1), \frac{z a+3 a+z+1}{4}, \frac{(z a+5 a+z+1)(z a+4 a+z+2)}{18 a+12 z a+6+12 z},\right.} \\
& \frac{(z a+z+2+6 a)(z a+z+1+7 a)(z a+z+3+5 a)}{16(2 z a+5 a+1+2 z)(z a+2 a+z+1)}, \\
& \left.\frac{(z a+z+4+6 a)(z a+z+1+9 a)(z a+z+3+7 a)(z a+z+2+8 a)}{20(2 z a+2 z+3+5 a)(z a+3 a+z+1)(2 z a+2 z+1+7 a)}\right]
\end{aligned}
$$


Theorem 6.8. We have

$$
\begin{gathered}
\sum_{T \in \mathcal{B}(n)} \prod_{h \in \mathcal{H}(T)} \frac{\prod_{i=1}^{h-1}(z a+z+(2 h-i) a+i)}{2 h \prod_{i=1}^{h-2}(2 z a+2 z+(2 h-2-i) a+i)} \\
=\frac{z(a+1)}{n !} \prod_{i=1}^{n-1}(z a+z+(2 n-i) a+i) .
\end{gathered}
$$

Proof. Let $f(x)$ be a power series in $x$ defined by

$$
f(x)=1+(a-1) x f(x)^{2 a /(a-1)}
$$

and $U_{n}(z, a)$ be the right-hand side of (6.16). Let $g(x)=f(x)-1$, then

$$
g(x)=(a-1) x(g(x)+1)^{2 a /(a-1)} .
$$

By the Lagrange inversion formula we have

$$
\begin{aligned}
{\left[x^{n}\right](g(x)+1)^{z} } & =\frac{1}{n}\left[x^{n-1}\right]\left(z(x+1)^{z-1}(a-1)^{n}(x+1)^{2 a n /(a-1)}\right) \\
& =\frac{z(a-1)^{n}}{n}\left[x^{n-1}\right](x+1)^{z-1+2 a n /(a-1)} \\
& =\frac{z(a-1)^{n}}{n(n-1) !} \prod_{i=0}^{n-2}\left(z-1+\frac{2 a n}{a-1}-i\right) . \\
& =\frac{z(a-1)}{n !} \prod_{i=1}^{n-1}(z(a-1)+2 a n-i(a-1))
\end{aligned}
$$

so that

$$
\left[x^{n}\right] f(x)^{z((a+1) /(a-1)}=\frac{z(a+1)}{n !} \prod_{i=1}^{n-1}(z(a+1)+2 a n-i(a-1))=U_{n}(z, a) .
$$

By (6.3) we have

$$
\rho(n)=\frac{\left[x^{n}\right] f(x)^{z(a+1) /(a-1)}}{\left[x^{n-1}\right] f(x)^{2 z(a+1) /(a-1)}}=\frac{U_{n}(z, a)}{U_{n-1}(2 z, a)} .
$$

Remark. Theorem 6.8 unifies a lot of formulas, including the two classical hook formulas (6.4) and (6.5), Postnikov's formula (6.7) and the generalization due to Lascoux, Du and Liu, another generalization of Postnikov's formula (6.10), Theorems 6.6 and 6.7 . 
$>\operatorname{hookexp}(\tan (\mathrm{x})+\sec (\mathrm{x}), 8)$;

$$
\left[1, \frac{1}{4}, \frac{1}{6}, \frac{1}{8}, \frac{1}{10}, \frac{1}{12}, \frac{1}{14}, \frac{1}{16}\right]
$$

$>\operatorname{hookexp}(z * \tan (x)+\sec (x), 8)$;

$$
\left[z, \frac{1}{4 z}, \frac{z}{3+3 z^{2}}, \frac{1}{8 z}, \frac{z}{5+5 z^{2}}, \frac{1}{12 z}, \frac{z}{7+7 z^{2}}, \frac{1}{16 z}\right]
$$

Theorem 6.9. We have

$$
\sum_{T \in \mathcal{B}} x^{|T|} \prod_{h \in \mathcal{H}(T), h \geq 2} \frac{1}{2 h}=\tan (x)+\sec (x)
$$

and

$$
\sum_{T \in \mathcal{B}} x^{|T|} \prod_{h \in \mathcal{H}(T)} \rho(h)=z \tan (x)+\sec (x)
$$

where

$$
\rho(n)= \begin{cases}z, & \text { if } n=1 \\ \frac{1}{2 n z}, & \text { if } n \text { is even } \\ \frac{z}{n\left(1+z^{2}\right)}, & \text { if } n \geq 3 \text { is odd }\end{cases}
$$

Proof. Let $f(x)=z \tan (x)+\sec (x)$. Then

$$
f^{2}(x)=\frac{1+z^{2} \sin ^{2}(x)}{\cos ^{2}(x)}+\frac{2 z \sin (x)}{\cos ^{2}(x)} .
$$

It is easy to verify that $\rho(1)=z$. For each $k \geq 1$,

$$
\begin{aligned}
{\left[x^{2 k}\right] f^{2}(x) } & =\left[x^{2 k}\right] \frac{1+z^{2} \sin ^{2}(x)}{\cos ^{2}(x)}-1=\left[x^{2 k}\right] \frac{1+z^{2} \sin ^{2}(x)-\cos ^{2}(x)}{\cos ^{2}(x)} \\
& =\left(1+z^{2}\right)\left[x^{2 k}\right] \tan ^{2}(x)=\frac{1+z^{2}}{z}\left[x^{2 k}\right] z \tan ^{2}(x) \\
& \left.=\frac{1+z^{2}}{z}\left[x^{2 k}\right](z \tan (x))^{\prime}-z\right) \\
& =\frac{1+z^{2}}{z}(2 k+1)\left[x^{2 k+1}\right](z \tan (x)) .
\end{aligned}
$$

Thus

$$
\rho(2 k+1)=\frac{\left[x^{2 k+1}\right] f(x)}{\left[x^{2 k}\right] f^{2}(x)}=\frac{z}{(2 k+1)\left(1+z^{2}\right)} .
$$


On the other hand,

$$
\left[x^{2 k-1}\right] \frac{2 z \sin (x)}{\cos ^{2}(x)}=2 z\left[x^{2 k-1}\right] \sec (x)^{\prime}=2 z(2 k)\left[x^{2 k}\right] \sec (x) .
$$

Hence

$$
\rho(2 k)=\frac{\left[x^{2 k}\right] f(x)}{\left[x^{2 k-1}\right] f^{2}(x)}=\frac{1}{2 z(2 k)} . \quad \square
$$

Remark. Formula (6.17) has a combinatorial interpretation due to Foata, Schützenberger and Strehl [FS73; FS74; Vi81; FH01] by using the model of André permutations. Note the difference with Theorem 7.1.

$>\operatorname{hookexp}\left((1+x) /\left(1+x^{\wedge} 2\right), 9\right)$;

(M.6.10)

$$
\left[1, \frac{-1}{2}, 1, \frac{-1}{4}, 1, \frac{-1}{6}, 1, \frac{-1}{8}, 1\right]
$$

Theorem 6.10. We have

$$
\sum_{T \in \mathcal{B}} x^{|T|} \prod_{h \in \mathcal{H}(T), h \text { even }} \frac{-1}{h}=\frac{1+x}{1+x^{2}} .
$$

Proof. Let

$$
f(x)=\frac{1}{1+x^{2}}+\frac{x}{1+x^{2}}
$$

Then

$$
f(x)^{2}=\frac{1}{1+x^{2}}+\frac{2 x}{\left(1+x^{2}\right)^{2}}
$$

We have

$$
\left[x^{2 k-1}\right] \frac{2 x}{\left(1+x^{2}\right)^{2}}=-\left[x^{2 k-1}\right]\left(\frac{1}{1+x^{2}}\right)^{\prime}=-(2 k)\left[x^{2 k}\right] \frac{1}{1+x^{2}},
$$

so that

$$
\rho(2 k)=\frac{\left[x^{2 k}\right] f(x)}{\left[x^{2 k-1}\right] f^{2}(x)}=-\frac{1}{2 k}
$$

and

$$
\rho(2 k+1)=\frac{\left[x^{2 k+1}\right] f(x)}{\left[x^{2 k}\right] f^{2}(x)}=1 .
$$

$>\operatorname{hookexp}\left((1+x) /\left(1+x^{\wedge} 3\right), 12\right)$;

$$
\left[1,0,-1, \frac{1}{2}, 0, \frac{-1}{2}, \frac{1}{3}, 0, \frac{-1}{3}, \frac{1}{4}, 0, \frac{-1}{4}\right]
$$


Theorem 6.11. We have

$$
\sum_{T \in \mathcal{B}} x^{|T|} \prod_{h \in \mathcal{H}(T)} \rho(h)=\frac{1+x}{1+x^{3}}
$$

where

$$
\rho(n)= \begin{cases}1 / k ; & \text { if } n=3 k-2 \\ 0 ; & \text { if } n=3 k-1 \\ -1 / k ; & \text { if } n=3 k\end{cases}
$$

Proof. Let

$$
f(x)=\frac{1}{1+x^{3}}+\frac{x}{1+x^{3}} .
$$

Then

$$
f(x)^{2}=\frac{1}{\left(1+x^{3}\right)^{2}}+\frac{2 x}{\left(1+x^{3}\right)^{2}}+\frac{x^{2}}{\left(1+x^{3}\right)^{2}} .
$$

Thus

$$
\left[x^{3 k-1}\right] \frac{x^{2}}{\left(1+x^{3}\right)^{2}}=-\frac{1}{3}\left[x^{3 k-1}\right]\left(\frac{x}{1+x^{3}}\right)^{\prime}=-k\left[x^{3 k}\right] \frac{1}{1+x^{3}} .
$$

It is easy to see that $\rho(3 k-1)=0$ and

$$
\rho(3 k)=\frac{\left[x^{3 k}\right] f(x)}{\left[x^{3 k-1}\right] f^{2}(x)}=-\frac{1}{k} .
$$

On the other hand,

$$
\left[x^{3 k-2}\right] \frac{x}{1+x^{3}}=\left[x^{3 k-3}\right] \frac{1}{1+x^{3}}=-\left[x^{3 k}\right] \frac{1}{1+x^{3}}
$$

and by (6.25)

$$
\left[x^{3 k-3}\right] \frac{1}{\left(1+x^{3}\right)^{2}}=\left[x^{3 k-1}\right] \frac{x^{2}}{\left(1+x^{3}\right)^{2}}=-k\left[x^{3 k}\right] \frac{1}{1+x^{3}} .
$$

Finally,

$$
\rho(3 k-2)=\frac{\left[x^{3 k-2}\right] f(x)}{\left[x^{3 k-3}\right] f^{2}(x)}=\frac{1}{k} .
$$

Consider the weight function $\rho$ that counts the leaves of binary trees.

$>[1, \operatorname{seq}(2, i=1.7)]$;

$$
[1,2,2,2,2,2,2,2]
$$

$>$ hookgen $(\%)$;

$$
1+x+4 x^{2}+18 x^{3}+88 x^{4}+456 x^{5}+2464 x^{6}+13736 x^{7}+78432 x^{8}
$$

The above generating function corresponds to the sequence A068764 in the on-line Encyclopedia of Integer Sequences [Slo]. It is equal to the generating function for the generalized Catalan numbers. 
Theorem 6.12. We have

$$
\sum_{\lambda \in \mathcal{B}} x^{|\lambda|} \prod_{h \in \mathcal{H}(\lambda), h \geq 2} 2=\frac{1-\sqrt{1-8 x(1-x)}}{4 x} .
$$

More generally,

$>[z, \operatorname{seq}(1, i=1 . .7)]$

$$
[z, 1,1,1,1,1,1,1]
$$

$>\operatorname{hookgen}(\%)$;

$$
\begin{aligned}
& 1+(z) x+(2 z) x^{2}+\left(2 z+z^{2}\right) x^{3}+\left(3 z+2 z^{2}\right) x^{4}+\left(2 z+5 z^{2}\right) x^{5} \\
& +\left(4 z+6 z^{2}+z^{3}\right) x^{6}+\left(2 z+11 z^{2}+2 z^{3}\right) x^{7}+\left(4 z+13 z^{2}+5 z^{3}\right) x^{8}
\end{aligned}
$$

The coefficient of $x^{n} z^{j}$ in the above generating function is the number of binary trees with $n$ vertices and $j$ leaves, which is given by the following formulas [Pr96].

Theorem 6.13 [Prodinger]. We have

$$
\sum_{\lambda \in \mathcal{B}} x^{|\lambda|} \prod_{h \in \mathcal{H}(\lambda), h=1} z=\sum_{n \geq 0, j \geq 1} A_{n, j} x^{n} z^{j}
$$

where

$$
A_{n, j}=\frac{2^{n+1-2 j}(n-1) !}{j !(j-1) !(n+1-2 j) !} .
$$

\section{Hook length formulas for complete binary trees}

A complete binary tree $T$ is a binary tree such that the two subtrees of each vertex $u$ are, either both empty, or both non-empty, except when $u$ is the latest vertex in the so-called inorder [Kn98a,p.319; Vi81]. In the latter case, the right subtree (resp. left subtree) of $u$ is empty (resp. non-empty) when $|T|$ is even. For example, there are five complete binary trees with $n=7$ vertices.
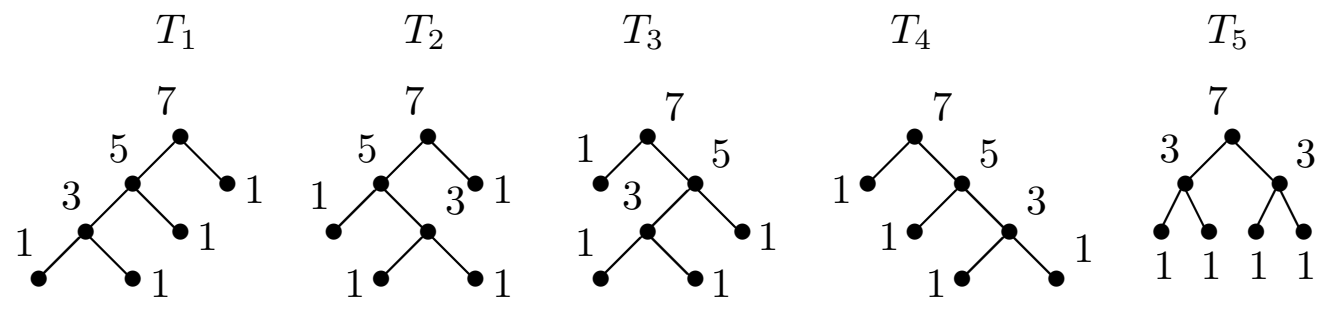

We have the hook length multi-sets $\mathcal{H}\left(T_{1}\right)=\mathcal{H}\left(T_{2}\right)=\mathcal{H}\left(T_{3}\right)=\mathcal{H}\left(T_{4}\right)=\{1,1,1,1,3,5$, $7\}$ and $\mathcal{H}\left(T_{5}\right)=\{1,1,1,1,3,3,7\}$. There are also five complete binary trees with $n=6$ vertices. 

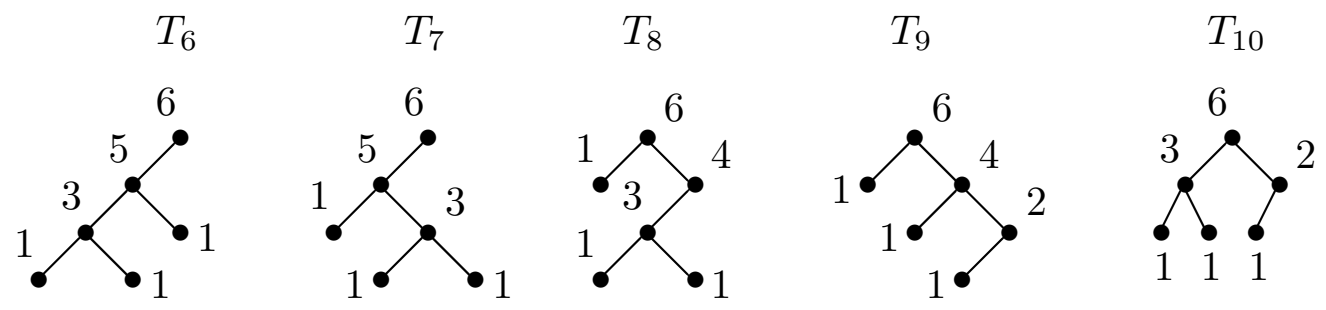

We have $\mathcal{H}\left(T_{6}\right)=\mathcal{H}\left(T_{7}\right)=\{1,1,1,3,5,6\}, \mathcal{H}\left(T_{8}\right)=\{1,1,1,3,4,6\}, \mathcal{H}\left(T_{9}\right)=$ $\{1,1,1,2,4,6\}$ and $\mathcal{H}\left(T_{10}\right)=\{1,1,1,2,3,6\}$.

Let $\mathcal{C}($ resp. $\mathcal{C}(n))$ denote the set of all complete binary trees (resp. all complete binary trees with $n$ vertices), so that

$$
\mathcal{C}=\bigcup_{n \geq 0} \mathcal{C}(n)
$$

Again, define the hook length expansion for complete binary trees by

$$
\sum_{T \in \mathcal{C}} x^{|T|} \prod_{h \in \mathcal{H}(T)} \rho(h)=f(x),
$$

where $f(x) \in K[[x]]$ is a power series in $x$ with coefficients in $K$ such that $f(0)=1$. See Sections 5 and 6 for more comments about the hook length expansion. Let $f(x)=1+f_{1} x+f_{2} x^{2}+f_{3} x^{3}+\cdots$ be the generating function for complete binary trees by the weight function $\rho$. With each $T \in \mathcal{C}(n)(n \geq 1)$ we can associate a triplet $\left(T^{\prime}, T^{\prime \prime}, v\right)$, where $T^{\prime} \in \mathcal{C}(k)(0 \leq k \leq n-1$ and $k$ is an odd integer $), T^{\prime \prime} \in \mathcal{C}(n-1-k)$ and the root $v$ of $T$ whose hook length $h_{v}=n$. Hence, (7.1) is equivalent to

$$
\rho(n) \sum_{k=0, k \text { odd }}^{n-1} f_{k} f_{n-1-k}=f_{n} \quad(n \geq 1) .
$$

Formula (7.2) can be used to calculate $f(x)$ for a given $\rho$, or to calculate $\rho$ for a given $f(x)$. It also has the equivalent form

$$
\rho(n)=\frac{\left[x^{n}\right] f(x)}{\left[x^{n-1}\right](f(x)-f(-x)) f(x) / 2},
$$

because

$$
\sum_{k \geq 1, k \text { odd }} f_{k} x^{k}=(f(x)-f(-x)) / 2 .
$$

Next we use the maple package HookExp to find hook formulas for complete binary trees, whose proofs are always based on (7.3). 


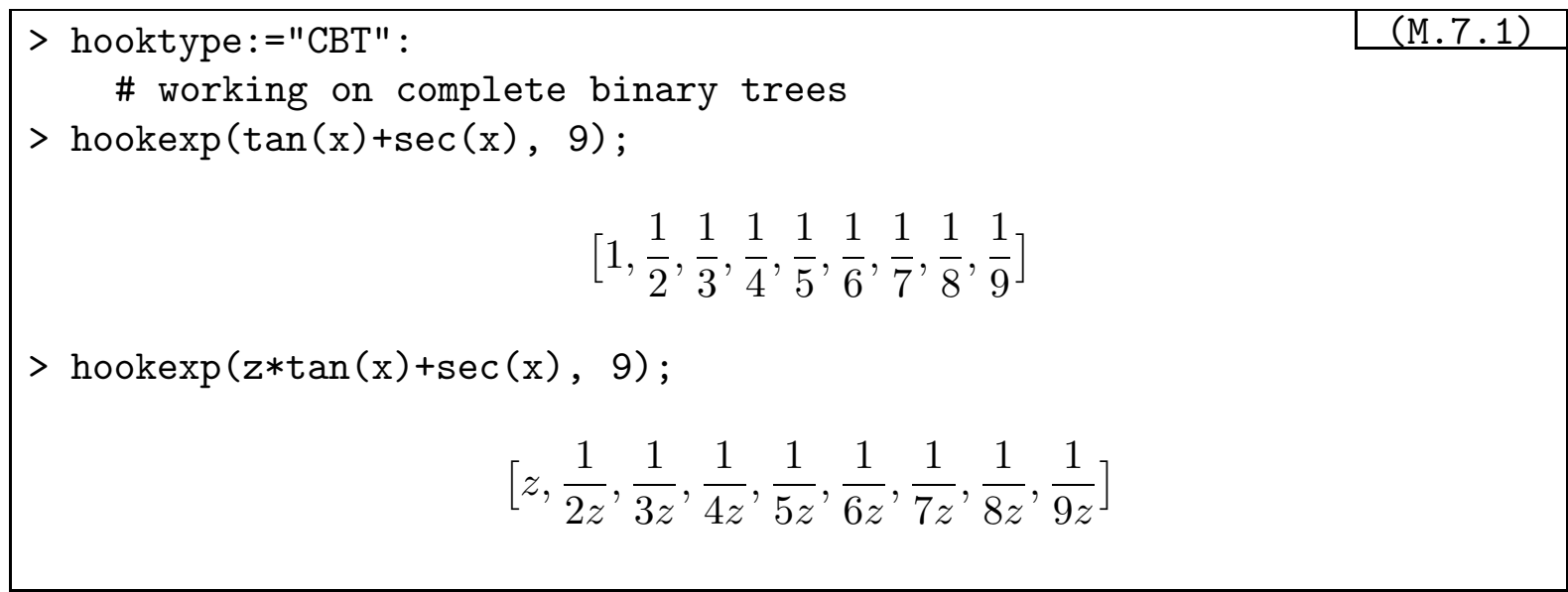

Theorem 7.1. We have

$$
\begin{gathered}
\sum_{T \in \mathcal{C}} x^{|T|} \prod_{h \in \mathcal{H}(T)} \frac{1}{h}=\tan (x)+\sec (x) . \\
\sum_{T \in \mathcal{C}} x^{|T|} \prod_{h \in \mathcal{H}(T), h=1} z \prod_{h \in \mathcal{H}(T), h \geq 2} \frac{1}{z h}=z \tan (x)+\sec (x) .
\end{gathered}
$$

Proof. By (7.3)

$$
\begin{aligned}
\rho(n) & =\frac{\left[x^{n}\right] z \tan (x)+\sec (x)}{\left[x^{n-1}\right] z \tan (x)(z \tan (x)+\sec (x))} \\
& =\frac{\left[x^{n}\right] z \tan (x)+\sec (x)}{z\left[x^{n-1}\right](z \tan (x)+\sec (x))^{\prime}-z} \\
& =\frac{\left[x^{n}\right] \tan (x)+\sec (x)}{z\left[x^{n}\right] n(\tan (x)+\sec (x))}=\frac{1}{z n} \cdot \quad
\end{aligned}
$$

Remark. Recall that the $n$-th Euler number is the coefficient of $x^{n} / n$ ! in the expansion of the series $\tan (x)+\sec (x)$ (see, e.g. [Vi81]). It is well-known that $E_{n}$ is equal to the number of alternating permutations of order $n$, which, in turn, is equal to the number of increasing labeled complete binary trees (See Theorem 6.1), so that

$$
\sum_{T \in \mathcal{C}(n)} n ! \prod_{v \in T} \frac{1}{h_{v}}=E_{n} .
$$

This gives a combinatorial proof of Theorem 7.1. Note that Theorem 6.9 also involves the Euler numbers, but the combinatorial argument is totally different.

\begin{tabular}{c}
$>\operatorname{hookexp}(\exp (\mathrm{x}), 9) ;$ \\
$\qquad\left[1, \frac{1}{2}, \frac{1}{6}, \frac{1}{16}, \frac{1}{40}, \frac{1}{96}, \frac{1}{224}, \frac{1}{512}, \frac{1}{1152}\right]$ \\
\hline
\end{tabular}


Theorem 7.2. We have

$$
\sum_{T \in \mathcal{C}} x^{|T|} \prod_{h \in \mathcal{H}(T), h \geq 2} \frac{1}{h 2^{h-2}}=e^{x} .
$$

Proof. From (7.3)

$$
\begin{aligned}
\rho(n) & =\frac{\left[x^{n}\right] e^{x}}{\left[x^{n-1}\right]\left(e^{x}-e^{-x}\right) e^{x} / 2}=\frac{2\left[x^{n}\right] e^{x}}{\left[x^{n-1}\right] e^{2 x}-1} \\
& =\frac{2 / n !}{2^{n-1} /(n-1) !}=\frac{1}{n 2^{n-2}} \cdot \quad \square
\end{aligned}
$$

Remark. We do not have any combinatorial proof of Theorem 7.2. See also [Ha08b]. It can be viewed as a complete binary tree version of Theorem 6.3.

$>\operatorname{hookexp}(1 /(1-x), 14)$;

(M.7.3)

$$
\left[1,1,1, \frac{1}{2}, \frac{1}{2}, \frac{1}{3}, \frac{1}{3}, \frac{1}{4}, \frac{1}{4}, \frac{1}{5}, \frac{1}{5}, \frac{1}{6}, \frac{1}{6}, \frac{1}{7}\right]
$$

Theorem 7.3. We have

$$
\sum_{T \in \mathcal{C}} x^{|T|} \prod_{h \in \mathcal{H}(T), h \geq 2} \rho(h)=\frac{1}{1-x},
$$

where

$$
\rho(n)= \begin{cases}1, & \text { if } n=1 \\ 1 / k, & \text { if } n=2 k+1 \quad(k \geq 1) \\ 1 / k, & \text { if } n=2 k(k \geq 1)\end{cases}
$$

Proof. Since

$$
\frac{x}{(1-x)\left(1-x^{2}\right)}=\frac{x+x^{2}}{\left(1-x^{2}\right)^{2}}=\left(x+x^{2}\right) \sum_{k \geq 0}(k+1) x^{2 k},
$$

we have

$$
\left[x^{2 k}\right] \frac{x}{(1-x)\left(1-x^{2}\right)}=\left[x^{2 k-1}\right] \frac{x}{(1-x)\left(1-x^{2}\right)}=k .
$$

By $(7.3)$

$$
\begin{aligned}
\rho(n) & =\frac{\left[x^{n}\right] 1 /(1-x)}{\left[x^{n-1}\right](1 /(1-x)-1 /(1+x)) /(1-x) / 2} \\
& \left.=\frac{1}{\left[x^{n-1}\right] x /(1-x) /\left(1-x^{2}\right)} \cdot \quad\right]
\end{aligned}
$$


Remark. For each complete binary tree $T$ of $2 k$ or $2 k+1$ vertices we obtain, in a bijective manner, a binary tree $T^{\prime}$ of $k$ vertices by deleting all leaves of $T$ [Kn98a, p.399]. This gives a combinatorial proof of Theorem 7.3 via Theorem 7.1.

$>$ hookexp $\left(\left(1-\operatorname{sqrt}\left(1-4 * \mathrm{x}^{\wedge} 2\right)\right) /\left(2 * \mathrm{x}^{\wedge} 2\right) *(1+\mathrm{x}), 9\right) ;$
$[1,1,1,1,1,1,1]$
$>\operatorname{hookexp}\left(\left(1-\operatorname{squt}\left(1-4 * \mathrm{x}^{\wedge} 2\right)\right) /\left(2 * \mathrm{x}^{\wedge} 2\right) *(1+\mathrm{z} * \mathrm{x}), 9\right) ;$
$\left[z, \frac{1}{z}, \frac{1}{z}, \frac{1}{z}, \frac{1}{z}, \frac{1}{z}, \frac{1}{z}\right]$

Theorem 7.4. We have

$$
\sum_{T \in \mathcal{C}} x^{|T|} \prod_{h \in \mathcal{H}(T)} 1=\frac{1-\sqrt{1-4 x^{2}}}{2 x^{2}}(1+x)
$$

and

$$
\sum_{T \in \mathcal{C}} x^{|T|} \prod_{h \in \mathcal{H}(T), h=1} z \prod_{h \in \mathcal{H}(T), h \geq 2} \frac{1}{z}=\frac{1-\sqrt{1-4 x^{2}}}{2 x^{2}}(1+z x)
$$

Proof. Let $f(x)$ be the right-hand side of (7.10). We can verify

$$
\begin{gathered}
\frac{(f(x)-f(-x)) f(x)}{2} \times \frac{x}{z}=f(x)-1-z x . \\
\rho(n)=\frac{\left[x^{n}\right] f(x)}{\left[x^{n-1}\right](f(x)-f(-x)) f(x) / 2}=\frac{1}{z} .
\end{gathered}
$$

Remark. The bijection between binary trees and complete binary trees described in Theorem 7.3 gives a combinatorial proof of Theorem 7.4 via Theorem 6.2.

$$
\begin{aligned}
& >\operatorname{hookexp}\left((1+\mathrm{x}) /\left(1+\mathrm{x}^{\wedge} 2\right), 11\right) ; \\
& \left.\qquad 1,-1,-1, \frac{-1}{2}, \frac{-1}{2}, \frac{-1}{3}, \frac{-1}{3}, \frac{-1}{4}, \frac{-1}{4}, \frac{-1}{5}, \frac{-1}{5}\right]
\end{aligned}
$$

Theorem 7.5. We have

$$
\sum_{T \in \mathcal{C}} x^{|T|} \prod_{h \in \mathcal{H}(T), h \geq 2} \rho(h)=\frac{1+x}{1+x^{2}}
$$


where

$$
\rho(n)= \begin{cases}1, & \text { if } n=1 \\ -1 / k, & \text { if } n=2 k+1(k \geq 1) \\ -1 / k, & \text { if } n=2 k \quad(k \geq 1)\end{cases}
$$

Proof. Let $f(x)$ be the right-hand side of (7.11)

$$
f(x):=\frac{1+x}{1+x^{2}}=(1+x) \sum_{k \geq 0}(-1)^{k} x^{2 k}
$$

and

$$
F(x):=\frac{(f(x)-f(-x)) f(x)}{2}=\frac{x+x^{2}}{\left(1+x^{2}\right)^{2}}=\left(x+x^{2}\right) \sum_{k \geq 0}(k+1)(-1)^{k} x^{2 k}
$$

We have

$$
\begin{aligned}
{\left[x^{2 k+1}\right] f(x) } & =\left[x^{2 k}\right] f(x)=(-1)^{k} \\
{\left[x^{2 k}\right] F(x) } & =\left[x^{2 k-1}\right] F(x)=(-1)^{k-1} k .
\end{aligned}
$$

By (7.3)

$$
\rho(n)=\frac{\left[x^{n}\right] f(x)}{\left[x^{n-1}\right] F(x)}=-\frac{1}{k} . \quad \square
$$

$>\operatorname{hookexp}\left((1+\mathrm{x}) /\left(1+\mathrm{x}^{\wedge} 4\right), 4\right)$;

Denominator is zero, no solution for $n=4$.

$>\operatorname{hookexp}\left((1+x) /\left(1+x^{\wedge} 3\right), 16\right)$;

$$
\left[1,0,-1,1,0,-1,1,0, \frac{-1}{2}, \frac{1}{2}, 0, \frac{-1}{2}, \frac{1}{2}, 0, \frac{-1}{3}, \frac{1}{3}\right]
$$

Theorem 7.6. We have

$$
\sum_{T \in \mathcal{C}} x^{|T|} \prod_{h \in \mathcal{H}(T), h \geq 2} \rho(h)=\frac{1+x}{1+x^{3}}
$$

where

$$
\rho(n)= \begin{cases}1, & \text { if } n=1 \\ 0, & \text { if } n=3 k-1(k \geq 1) \\ -1 / k, & \text { if } n=6 k-3 \text { or } n=6 k(k \geq 1) \\ 1 / k, & \text { if } n=6 k-2 \text { or } n=6 k+1(k \geq 1)\end{cases}
$$


Proof. Let $f(x)$ be the right-hand side of (7.13)

$$
f(x):=\frac{1+x}{1+x^{3}}=(1+x) \sum_{k \geq 0}(-1)^{k} x^{3 k}
$$

and

$$
\begin{aligned}
F(x) & :=\frac{(f(x)-f(-x)) f(x)}{2} \\
& =\frac{x+x^{2}-x^{3}-2 x^{4}-x^{5}+x^{6}+x^{7}}{\left(1-x^{6}\right)^{2}} \\
& =\left(x+x^{2}-x^{3}-2 x^{4}-x^{5}+x^{6}+x^{7}\right) \sum_{k \geq 0}(k+1) x^{6 k} .
\end{aligned}
$$

We have

$$
\begin{aligned}
{\left[x^{3 k+2}\right] f(x) } & =0 ; \\
{\left[x^{3 k+1}\right] f(x) } & =\left[x^{3 k}\right] f(x)=(-1)^{k} ; \\
{\left[x^{6 k+2}\right] F(x) } & =\left[x^{6 k+6}\right] F(x)=k+1 ; \\
{\left[x^{6 k+3}\right] F(x) } & =\left[x^{6 k+5}\right] F(x)=-(k+1) ; \\
{\left[x^{6 k+4}\right] F(x) } & =-2(k+1) .
\end{aligned}
$$

By $(7.3)$

$$
\rho(n)=\frac{\left[x^{n}\right] f(x)}{\left[x^{n-1}\right] F(x)} .
$$

\section{Hook length formulas for Fibonacci trees}

A Fibonacci tree $T$ is a binary tree such that the right subtree of each vertex $u$ is either an empty tree, or a binary tree with only one vertex [St75, SY89]. For example, there are five Fibonacci trees with $n=4$ vertices.
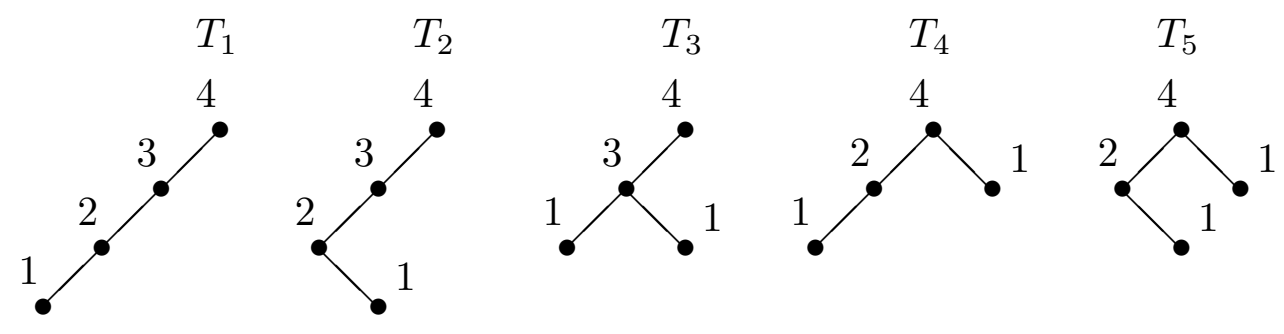

We have the hook length multi-sets $\mathcal{H}\left(T_{1}\right)=\mathcal{H}\left(T_{2}\right)=\{1,2,3,4\}, \mathcal{H}\left(T_{4}\right)=\mathcal{H}\left(T_{5}\right)=$ $\{1,1,2,4\}$ and $\mathcal{H}\left(T_{3}\right)=\{1,1,3,4\}$. 
Let $\mathcal{F}($ resp. $\mathcal{F}(n))$ denote the set of all Fibonacci trees (resp. all Fibonacci trees with $n$ vertices), so that

$$
\mathcal{F}=\bigcup_{n \geq 0} \mathcal{F}(n) .
$$

As for binary trees, we define the hook length expansion for Fibonacci trees by

$$
\sum_{T \in \mathcal{F}} x^{|T|} \prod_{h \in \mathcal{H}(T)} \rho(h)=f(x),
$$

where $f(x) \in K[[x]]$ is a power series in $x$ with coefficients in $K$ such that $f(0)=1$. See Sections 5 and 6 for more comments about the hook length expansion. Let $f(x)=1+f_{1} x+f_{2} x^{2}+f_{3} x^{3}+\cdots$ be the generating function for Fibonacci trees by the weight function $\rho$. By definition of Fibonacci trees formula (8.1) is equivalent to

$$
f_{n}=\rho(n) f_{n-1}+\rho(n) \rho(1) f_{n-2} .
$$

Formula (8.2) can be used to calculate $f(x)$ for a given $\rho$, or to calculate $\rho$ for a given $f(x)$. Next we use the maple package HookExp to find hook formulas for Fibonacci trees, whose proofs are always based on (8.2).

$>$ hooktype:="FT" \# working on Fibonacci trees
$>$ hookexp(1/(1-x-x^2),9);
\[ [1,1,1,1,1,1,1,1,1] \]

Theorem 8.1. We have

$$
\sum_{T \in \mathcal{F}} x^{|T|} \prod_{h \in \mathcal{H}(T)} 1=\frac{1}{1-x-x^{2}} .
$$

Proof. Let $f(x)=1+\sum_{n \geq 1} f_{n} x^{n}$ be the right-hand side of (8.3), then $f_{n}=$ $f_{n-1}+f_{n-2}$. Relation (8.2) is verified.

Remark. The number of Fibonacci trees with $n$ vertices is the $n$-th Fibonacci number.

$>\operatorname{hookexp}(\exp (\mathrm{x}), 9)$;

$$
\left[1, \frac{1}{4}, \frac{1}{9}, \frac{1}{16}, \frac{1}{25}, \frac{1}{36}, \frac{1}{49}, \frac{1}{64}, \frac{1}{81}\right]
$$


Theorem 8.2. We have

$$
\sum_{T \in \mathcal{F}} x^{|T|} \prod_{h \in \mathcal{H}(T)} \frac{1}{h^{2}}=e^{x}
$$

Proof. It suffices to verify relation (8.2).

$$
\frac{1}{n !}=\frac{1}{n^{2}} \frac{1}{(n-1) !}+\frac{1}{n^{2}} \frac{1}{(n-2) !} .
$$

Remark. The number of ordered pairs of increasing labeled Fibonacci trees on $\{1,2, \ldots, n\}$ of the same shape (i.e., the same Fibonacci tree) is equal to $n$ ! (See, e.g., [St75, SY89]).

$>$ hookexp $\left(\exp \left(x+x^{\wedge} 2 / 2\right), 9\right)$;

$$
\left[1, \frac{1}{2}, \frac{1}{3}, \frac{1}{4}, \frac{1}{5}, \frac{1}{6}, \frac{1}{7}, \frac{1}{8}, \frac{1}{9}\right]
$$

Theorem 8.3. We have

$$
\sum_{T \in \mathcal{F}} x^{|T|} \prod_{h \in \mathcal{H}(T)} \frac{1}{h}=\exp \left(x+x^{2} / 2\right) .
$$

Proof. Let $f(x)=1+\sum_{n \geq 1} f_{n} x^{n}$ be the left-hand side of (8.6). From (8.2) we have

$$
f_{n}=\frac{1}{n} f_{n-1}+\frac{1}{n} f_{n-2} .
$$

On the other hand, let $\sum_{n \geq 0} a_{n} x^{n} / n$ ! be right-hand side of (8.6). We know that $a_{n}$ is equal to the number of involutions of order $n$. Thus

$$
a_{n}=(n-1) a_{n-2}+a_{n-1} .
$$

Comparing (8.7) and (8.8) yields $f_{n}=a_{n} / n$ !

Remark. The number of increasing labeled Fibonacci trees on $[n]$ is equal to the number of involutions of order $n$ (see [St75, SY89]).

$>\operatorname{hookexp}(1 /(1-x), 9)$;

$$
\left[1, \frac{1}{2}, \frac{1}{2}, \frac{1}{2}, \frac{1}{2}, \frac{1}{2}, \frac{1}{2}, \frac{1}{2}, \frac{1}{2}\right]
$$


Theorem 8.4. We have

$$
\sum_{T \in \mathcal{F}} x^{|T|} \prod_{h \in \mathcal{H}(T), h \geq 2} \frac{1}{2}=\frac{1}{1-x}
$$

Proof. We check relation (8.2):

$$
1=\frac{1}{2}+\frac{1}{2}
$$

$>$ hookexp(1/(1-x)^z, 6): $\operatorname{map}($ factor, $\%)$;

$$
\left[z, \frac{1+z}{4}, \frac{(z+2)(1+z)}{9 z+3}, \frac{(z+3)(z+2)}{16 z+8}, \frac{(z+4)(z+3)}{25 z+15}, \frac{(z+5)(z+4)}{36 z+24}\right]
$$

Theorem 8.5. We have

$$
\sum_{T \in \mathcal{F}} x^{|T|} \prod_{h \in \mathcal{H}(T)} \frac{(h+z-1)(h+z-2)}{h(h z+h-2)}=\frac{1}{(1-x)^{z}}
$$

or

$$
\sum_{T \in \mathcal{F}(n)} \prod_{h \in \mathcal{H}(T)} \frac{(h+z-1)(h+z-2)}{h(h z+h-2)}=\left(\begin{array}{c}
n+z-1 \\
z-1
\end{array}\right) .
$$

Proof. We check relation (8.2):

$$
\left.\left(\begin{array}{c}
n+z-1 \\
z-1
\end{array}\right)=\frac{(n+z-1)(n+z-2)}{n(n z+n-2)}\left(\begin{array}{c}
n+z-2 \\
z-1
\end{array}\right)+z\left(\begin{array}{c}
n+z-3 \\
z-1
\end{array}\right)\right) .
$$

\footnotetext{
$>\operatorname{hookexp}((1-\operatorname{sqrt}(1-4 * x)) /(2 * x), 15)$;

$$
\left[1,1, \frac{5}{3}, 2, \frac{42}{19}, \frac{33}{14}, \frac{143}{58}, \frac{130}{51}, \frac{34}{13}, \frac{323}{121}, \frac{19}{7}, \frac{322}{117}, \frac{1150}{413}, \frac{45}{16}\right]
$$

$>$ guess $(\%)$;

$$
\frac{4(2 n-3)(2 n-1)}{(n+1)(5 n-6)}
$$
}


Theorem 8.6. We have

$$
\sum_{T \in \mathcal{F}} x^{|T|} \prod_{h \in \mathcal{H}(T), h \geq 2} \frac{4(2 h-1)(2 h-3)}{(h+1)(5 h-6)}=\frac{1-\sqrt{1-4 x}}{2 x}
$$

or

$$
\sum_{T \in \mathcal{F}(n)} \prod_{h \in \mathcal{H}(T), h \geq 2} \frac{4(2 h-1)(2 h-3)}{(h+1)(5 h-6)}=\frac{1}{n+1}\left(\begin{array}{c}
2 n \\
n
\end{array}\right) .
$$

Proof. We check relation (8.2):

$$
\frac{1}{n+1}\left(\begin{array}{c}
2 n \\
n
\end{array}\right)=\frac{4(2 n-1)(2 n-3)}{(n+1)(5 n-6)}\left(\frac{1}{n}\left(\begin{array}{c}
2 n-2 \\
n-1
\end{array}\right)+\frac{1}{n-1}\left(\begin{array}{c}
2 n-4 \\
n-2
\end{array}\right)\right) .
$$

$>\operatorname{hookexp}\left(((1-\operatorname{sqrt}(1-4 * x)) /(2 * x))^{\wedge} z, 7\right):$

$$
\begin{aligned}
& {\left[z, \frac{3+z}{4}, \frac{(z+5)(z+4)}{9 z+9}, \frac{(z+5)(z+7)(z+6)}{8(2 z+5)(z+2)}\right.} \\
& \left.\frac{(z+9)(z+8)(z+7)(z+6)}{5(5 z+14)(z+5)(3+z)}, \frac{(z+10)(z+9)(z+8)(z+11)}{36(z+4)(3+z)(z+6)}\right]
\end{aligned}
$$

$(\mathrm{M} .8 .7)$

Theorem 8.7. We have

$$
\sum_{T \in \mathcal{F}} x^{|T|} \prod_{h \in \mathcal{H}(T)} \rho(z ; n)=\left(\frac{1-\sqrt{1-4 x}}{2 x}\right)^{z}
$$

where

$$
\rho(z ; n)=\frac{(z+2 n-4)(z+2 n-3)(z+2 n-2)(z+2 n-1)}{n(z+n-2)(z+n)(n z+4 n-6)} .
$$

In other words,

$$
\sum_{T \in \mathcal{F}(n)} \prod_{h \in \mathcal{H}(T)} \rho(z ; n)=\frac{z}{n !} \prod_{i=1}^{n-1}(2 n-i+z) .
$$

Proof. As for the proof of Theorem 8.6, we check relation (8.2).

$>\operatorname{hookexp}\left(\left(1-\operatorname{sqrt}\left(1-4 * x^{\wedge} 2\right)\right) /\left(2 * x^{\wedge} 2\right) *(1+z * x), 11\right)$;

$$
\left[z, \frac{1}{2 z}, \frac{z}{1+z^{2}}, \frac{1}{z}, \frac{2 z}{z^{2}+2}, \frac{5}{4 z}, \frac{5 z}{2 z^{2}+5}, \frac{7}{5 z}, \frac{14 z}{5 z^{2}+14}, \frac{3}{2 z}\right]
$$


Theorem 8.8. We have

$$
\sum_{T \in \mathcal{F}} x^{|T|} \prod_{h \in \mathcal{H}(T)} \rho(h)=\frac{1-\sqrt{1-4 x^{2}}}{2 x^{2}}(1+z x),
$$

where

$$
\rho(n)= \begin{cases}z, & \text { if } n=1 ; \\ \frac{2 k-1}{(k+1) z}, & \text { if } n=2 k(k \geq 1) \\ \frac{2(2 k-1) z}{(k+1) z^{2}+2(2 k-1)}, & \text { if } n=2 k+1(k \geq 1) .\end{cases}
$$

In an equivalent manner, it means that

$$
\begin{aligned}
\sum_{T \in \mathcal{F}(2 k+1)} \prod_{h \in \mathcal{H}(T)} \rho(h) & =\frac{z}{k+1}\left(\begin{array}{c}
2 k \\
k
\end{array}\right) ; \\
\sum_{T \in \mathcal{F}(2 k)} \prod_{h \in \mathcal{H}(T)} \rho(h) & =\frac{1}{k+1}\left(\begin{array}{c}
2 k \\
k
\end{array}\right) .
\end{aligned}
$$

Proof. Relation (8.2) is being verified when $n$ is odd (resp. even)

$$
\begin{aligned}
& \frac{z}{k+1}\left(\begin{array}{c}
2 k \\
k
\end{array}\right)=\frac{2(2 k-1) z}{(k+1) z^{2}+2(2 k-1)}\left(\frac{1}{k+1}\left(\begin{array}{c}
2 k \\
k
\end{array}\right)+z \frac{z}{k}\left(\begin{array}{c}
2 k-2 \\
k-1
\end{array}\right)\right) \\
& \text { (resp. } \left.\frac{1}{k+1}\left(\begin{array}{c}
2 k \\
k
\end{array}\right)=\frac{2 k-1}{(k+1) z}\left(\frac{z}{k}\left(\begin{array}{c}
2 k-2 \\
k-1
\end{array}\right)+z \frac{1}{k}\left(\begin{array}{c}
2 k-2 \\
k-1
\end{array}\right)\right)\right) . \quad
\end{aligned}
$$

$>$ hookexp $\left((1+x) /\left(1+x^{\wedge} 2\right), 4\right)$;

Denominator is zero, no solution for $n=3$.

$>\operatorname{hookexp}\left((1+x) /\left(1+x^{\wedge} 3\right), 16\right)$;

$$
[1,0,-1,1,0,-1,1,0,-1,1,0,-1,1,0,-1,1]
$$

Theorem 8.9. We have

$$
\sum_{T \in \mathcal{F}} x^{|T|} \prod_{h \in \mathcal{H}(T)} \rho(h)=\frac{1+x}{1+x^{3}}
$$

where

$$
\rho(n)= \begin{cases}1, & \text { if } n \equiv 1 \bmod 3 \\ 0, & \text { if } n \equiv 2 \bmod 3 \\ -1, & \text { if } n \equiv 0 \bmod 3\end{cases}
$$


Proof. Let

$$
\sum_{n \geq 0} f_{n} x^{n}=\frac{1+x}{1+x^{3}}=\frac{1}{1-x+x^{2}} .
$$

We have $f_{3 k-1}=0, f_{3 k}=(-1) f_{3 k-2}$ and $f_{3 k-2}=f_{3 k-3}$. Relation (8.2) is then verified.

In fact, there is another generalization of Theorem 8.4. Consider the weight function $\rho$ that counts the leaves of Fibonacci trees.

$>$ > $\mathrm{z}, \operatorname{seq}(1, \mathrm{i}=1 . .6)] ; \quad[z, 1,1,1,1,1,1]$
$>\operatorname{hookgen}(\%) ;$
$\begin{aligned} & 1+(z) x+(2 z) x^{2}+\left(z^{2}+2 z\right) x^{3}+\left(3 z^{2}+2 z\right) x^{4}+\left(z^{3}+5 z^{2}+2 z\right) x^{5} \\ & \quad+\left(4 z^{3}+7 z^{2}+2 z\right) x^{6}+\left(z^{4}+9 z^{3}+9 z^{2}+2 z\right) x^{7}\end{aligned}$

The above generating function corresponds to the sequence A129710 in the on-line Encyclopedia of Integer Sequences [Slo] and is equal to the right-hand side of (8.19) below.

Theorem 8.10. We have

$$
\sum_{\lambda \in \mathcal{F}} x^{|\lambda|} \prod_{h \in \mathcal{H}(\lambda), h=1} z=\frac{1+(z-1) x}{1-x-z x^{2}} .
$$

Acknowledgements. The author wishes to thank Dominique Foata for helpful discussions during the preparation of this paper. He also thanks the referee who made knowledgeable remarks that have been taken into account in the final version.

\section{References}

[An76] Andrews, George E., The Theory of Partitions, Addison-Wesley, Reading, 1976 (Encyclopedia of Math. and Its Appl., vol. 2).

[Be98] Bessenrodt, Christine, On hooks of Young diagrams, Ann. of Comb., 2 (1998), pp. 103-110.

[BFS92] Bergeron, François; Flajolet, Philippe; Salvy, Bruno, Varieties of increasing trees, Lecture Notes in Comput. Sci., 581, Springer, Berlin, 1992.

[BG04] Béraud, Jean-François; Gauthier, Bruno, Maple package to guess closed form for a sequence of numbers, http://www-igm.univ-mlv.fr/〜gauthier/GUESS.html, see also [Kr01]. 
[BM02] Bacher, Roland; Manivel, Laurent, Hooks and Powers of Parts in Partitions, Sém. Lothar. Combin., vol. 47, article B47d, 2001, 11 pages.

[CY08] Chen, William Y.C.; Yang, Laura L.M., On Postnikov's hook length formula for binary trees, European Journal of Combinatorics, 29 (2008), pp. 1563-1565.

[DL08] Du, Rosena R. X.; Liu, Fu, $(k, m)$-Catalan Numbers and Hook Length Polynomials for Plane Trees, European J. Combin, 28 (2007), pp. 1312-1321.

[Dy72] Dyson, Freeman J., Missed opportunities, Bull. Amer. Math. Soc., 78 (1972), pp. 635-652.

[Eu83] Euler, Leonhard, The expansion of the infinite product $(1-x)(1-x x)\left(1-x^{3}\right)(1-$ $\left.x^{4}\right)\left(1-x^{5}\right)\left(1-x^{6}\right)$ etc. into a single series, English translation from the Latin by Jordan Bell on arXiv:math.HO/0411454.

[FH01] Foata, Dominique; Han, Guo-Niu, Arbres minimax et polynômes d'André, Advances in Appl. Math., 27 (2001), pp. 367-389.

[FS73] Foata, Dominique; Schützenberger, Marcel-Paul, Nombres d'Euler et permutations alternantes, A survey of Combinatorial Theory J.N. Srivastava et al., eds., p. 173-187. Amsterdam, North-Holland, 1973.

[FS74] Foata, Dominique; Strehl, Volker, Rearrangements of the symmetric group and enumerative properties of the tangent and secant numbers, Math. Zeitschrift, 137 (1974), pp. 257-264.

[FRT54] Frame, J. Sutherland; Robinson, Gilbert de Beauregard; Thrall, Robert M., The hook graphs of the symmetric groups, Canadian J. Math., 6 (1954), pp. 316-324.

[Ga01] Garvan, Frank, A $q$-product Tutorial for a $q$-series Maple Package, The Andrews Festschrift. Seventeen Papers on Classical Number Theory and Combinatorics, D. Foata, G.-N. Han eds., Springer-Verlag, Berlin Heidelberg, 2001, pp. 111-138. Sem. Lothar. Combin. Art. B42d, 27 pp.

[GKS90] Garvan, Frank; Kim, Dongsu; Stanton, Dennis, Cranks and t-cores, Invent. Math., 101 (1990), pp. 1-17.

[GNW79] Greene, Curtis; Nijenhuis, Albert; Wilf, Herbert S., A probabilistic proof of a formula for the number of Young tableaux of a given shape, Adv. in Math., 31 (1979), pp. 104-109.

[GS06] Gessel, Ira M.; Seo, Seunghyun, A refinement of Cayley's formula for trees, Electron. J. Combin., 11(2004/06), no. 2, Research Paper 27, 23 pp.

[GV85] Gessel, Ira; Viennot, Gerard, Binomial determinants, paths, and hook length formulae, Adv. in Math., 58 (1985), pp. 300-321.

[Ha08a] Han, Guo-Niu, An explicit expansion formula for the powers of the Euler Product in terms of partition hook lengths, arXiv:0804.1849v2, Math.CO, 35 pages, 2008.

[Ha08b] Han, Guo-Niu, New hook length formulas for binary trees, Combinatorica, in press, 4 pages, 2008.

[Ha08c] Han, Guo-Niu, Yet another generalization of Postnikov's hook length formula for binary trees, SIAM J. Discrete Math, in press, 4 pages, 2008.

[Ha08d] Han, Guo-Niu, Some conjectures and open problems about partition hook length, Experimental Mathematics, in press, 12 pages, 2008. 
[Ha08e] Han, Guo-Niu, The Nekrasov-Okounkov hook length formula: refinement, elementary proof, extension and applications, in preparation, 28 pages, 2008.

[JK81] James, Gordon; Kerber, Adalbert, The representation theory of the symmetric group, Encyclopedia of Mathematics and its Applications, 16. Addison-Wesley Publishing, Reading, MA, 1981.

[JS89] Joichi, James T.; Stanton, Dennis, An involution for Jacobi's identity, Discrete Math., 73 (1989), pp. 261-271.

[Kn70] Knuth, Donald E., Permutations, matrices, and generalized Young tableaux, Pacific J. Math., 34 (1970), pp. 709-727.

[Kn98a] Knuth, Donald E., The Art of Computer Programming, vol. 1, Fundamental Algorithms, 3rd ed., Addison Wesley Longman, 1997.

[Kn98b] Knuth, Donald E., The Art of Computer Programming, vol. 3, Sorting and Searching, 2nd ed., Addison Wesley Longman, 1998.

[Kr01] Krattenthaler, Christian, RATE - A Mathematica guessing machine, http://

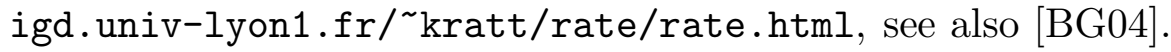

[Kr99] Krattenthaler, Christian, Another involution principle-free bijective proof of Stanley's hook-content formula, J. Combin. Theory Ser. A, 88 (1999), pp. 6692.

[La01] Lascoux, Alain, Symmetric Functions and Combinatorial Operators on Polynomials, CBMS Regional Conference Series in Mathematics, Number 99, 2001.

[Ma72] Macdonald, Ian G., Affine root systems and Dedekind's $\eta$-function, Invent. Math., 15 (1972), pp. 91-143.

[Ma95] Macdonald, Ian G., Symmetric Functions and Hall Polynomials, Second Edition, Clarendon Press, Oxford, 1995.

[MY07] Moon, J. W.; Yang, Laura L. M., Postnikov identities and Seo's formulas, Bull. Inst. Combin. Appl., 49 (2007), pp. 21-31.

[N006] Nekrasov, Nikita A.; Okounkov, Andrei, Seiberg-Witten theory and random partitions. The unity of mathematics, 525-596, Progr. Math., 244, Birkhaeuser Boston. 2006. (See also arXiv:hep-th/0306238v2, 90 pages, 2003).

[NPS97] Novelli, Jean-Christophe; Pak, Igor; Stoyanovskii, Alexander V., A direct bijective proof of the hook-length formula, Discrete Math. Theor. Comput. Sci., 1 (1997), pp. $53-67$.

[Po04] Postnikov, Alexander, Permutohedra, associahedra, and beyond, arXiv:math. CO/0507163, 2004.

[Pr96] Prodinger, Helmut, A Note on the Distribution of the Three Types of Nodes in Uniform Binary Trees, with comments by Christian Krattenthaler, Guo-Niu Han and Günter Rote, Séminaire Lotharingien de Combinatoire, Article B38b, 1996, 5 pp.

[RW83] Remmel, Jeffrey B.; Whitney, Roger A, bijective proof of the hook formula for the number of column strict tableaux with bounded entries, European J. Combin., 4 (1983), pp. 45-63.

[Sch76] Schützenberger, Marcel-Paul, La correspondance de Robinson, dans "Combinatoire et Représentation du Groupe Symétrique", Lecture Notes in Mathematics, 
Springer-Verlag, vol. 579, 1976, p. 59-113.

[Se08] Seo, Seunghyun, A combinatorial proof of Postnikov's identity and a generalized enumeration of labeled trees, arXiv:math.CO/0409323.

[Slo] Sloane, Neil; al., The On-Line Encyclopedia of Integer Sequences, http:// www.research.att. com/ njas/sequences/.

[St75] Stanley, Richard P., The Fibonacci lattice, Fibonacci Quart., 13 (1975), pp. 215232.

[St76] Stanley, Richard P., Theory and application of plane partitions (II), Studies in Appl. Math., 50 (1971), pp. 259-279.

[St97] Stanley, Richard P., Enumerative Combinatorics, vol. 1, Cambridge university press, 1997 .

[St99] Stanley, Richard P., Enumerative Combinatorics, vol. 2, Cambridge university press, 1999 .

[SY89] Sagan, Bruce E.; Yeh, Yeong Nan, Probabilistic algorithms for trees, Fibonacci Quart., 27 (1989), pp. 201-208.

[Vi81] Viennot, Gérard, Interprétations combinatoires des nombres d'Euler et de Genocchi, Séminaire de Théorie des Nombres, Bordeaux, 94 pages, 1981.

[Ze84] Zeilberger, Doron, A short hook-lengths bijection inspired by the Greene-Nijenhuis-Wilf proof, Discrete Math., 51 (1984), pp. 101-108. 